\title{
1 Enrichment of specific GABAergic neuronal types in the mouse perirhinal cortex \\ 2
}

\section{Authors}

4 Maximiliano José Nigro ${ }^{1 *}$, Kasper Kjelsberg ${ }^{1}$, Laura Convertino ${ }^{1,2}$, Rajeevkumar Raveendran Nair ${ }^{1}$, 5 Menno P. Witter ${ }^{1}$

$6{ }^{1}$ Kavli Institute for Systems Neuroscience, Center for Neural Computation, Egil and Pauline Braathen and Fred Kavli Center

7 for Cortical Microcircuits, NTNU, Trondheim, Norway

$8{ }^{2}$ Current address: Institute of Cognitive Neuroscience and Wellcome Centre for Human Neuroimaging, University College

9 London, London, United Kingdom

10

$11{ }^{*}$ Corresponding author

12 Maximiliano José Nigro

13 Maximiliano.j.nigro@ntnu.no

14

\section{Abstract}

16 GABAergic neurons represent $10-15 \%$ of the neuronal population of the cortex but exert a powerful 17 control over information flow in cortical circuits. GABAergic neurons show an extraordinary diversity in 18 their morphology, physiology, molecular markers and connectivity. This diversity allows GABAergic 19 neurons to participate in a wide variety of microcircuit motifs. The diversity of GABAergic neurons has 20 been shown to be conserved across cortical regions. The GABAergic population can be broadly divided 21 in three major classes parvalbumin, somatostatin and 5HT3aR groups. The largest GABAergic class in 22 the cortex is represented by the parvalbumin-expressing fast-spiking neurons, which provide powerful 23 somatic inhibition to their postsynaptic targets. Recently, the density of parvalbumin-expressing neurons 24 has been shown to be lower in associative areas of the mouse cortex, including the perirhinal cortex, as 25 compared to sensory and motor areas. In the present study we investigated whether this reduction in 26 parvalbumin-expressing neurons leads to a decreased GABAergic population, or to an enrichment of 27 other GABAergic cell-types. We found that the GABAergic population of the perirhinal cortex is 28 comparable to that of a primary sensory area, and it is enriched of neurons belonging to the $5 \mathrm{HT} 3 \mathrm{aR}$ 29 group. We also demonstrate that, despite the low density of parvalbumin-expressing neurons, the 30 perirhinal cortex contains a comparable population of fast-spiking neurons, most of which do not express 31 parvalbumin. Our results demonstrate a yet uncharacterized diversity within the fast-spiking population 32 across cortical regions.

33

\section{Introduction}

35 The wide neuronal diversity of the cerebral 36 cortex is thought to endow it with a variety of 37 circuit motifs that shape information processing 38 (Harris and Shepherd 2015; Luo 2021). The 39 cerebral cortex can be parcellated in functional 40 areas that show specific cytoarchitectonic 41 features and molecular marker expression (van 42 Essen and Glasser, 2018). The relationship 43 between cortical parcellation and distribution of 44 neuron types in the cortex is poorly understood. 45 GABAergic neurons represent $10-15 \%$ of the 46 neuronal population and their diversity allows 47 them to participate in different cortical 48 microcircuits (Fishell and Kepecs, 2014; 49 Tremblay et al., 2021). GABAergic neurons are 50 divided in two major groups according to 51 embryonal origin: those derived from the medial 52 ganglionic eminence (MGE) and those derived 53 from the caudal ganglionic eminence (CGE) 54 (Rudy et al 2011). MGE-derived GABAergic
55 neurons are further divided according to 56 molecular marker expression into parvalbumin 57 (PV)-expressing and somatostatin (SST)58 expressing interneurons (INs). CGE-derived 59 interneurons (CGE-INs) are labeled by GFP in 60 the 5HT3aR-GFP mouse line (Lee et al 2010). 61 Transcriptomic analysis has confirmed this 62 classification and further divided the CGE group 63 into three major classes: vasoactive intestinal 64 peptide (VIP)-expressing, Lamp5, and Sncg 65 groups (Tasic et al 2018). Molecularly defined 66 GABAergic neurons have been shown to be 67 homogeneously distributed across sensory68 motor areas of the neocortex ( $\mathrm{Xu}$ and Callaway 69 2010). However, a recent cortex-wide 70 examination of the distribution of three major 71 GABAergic groups (PV, SST, VIP) revealed 72 regional specializations (Whissell et al 2015; $73 \mathrm{Kim}$ et al 2017). In particular, the density of PV74 INs decreases in association areas, including 75 the perirhinal cortex (PER). PV-INs are the most 
76 abundant GABAergic type in the neocortex and 77 provide powerful perisomatic inhibition to their
78 postsynaptic targets (Tremblay et al., 2016). 79 PER is part of the parahippocampal region and
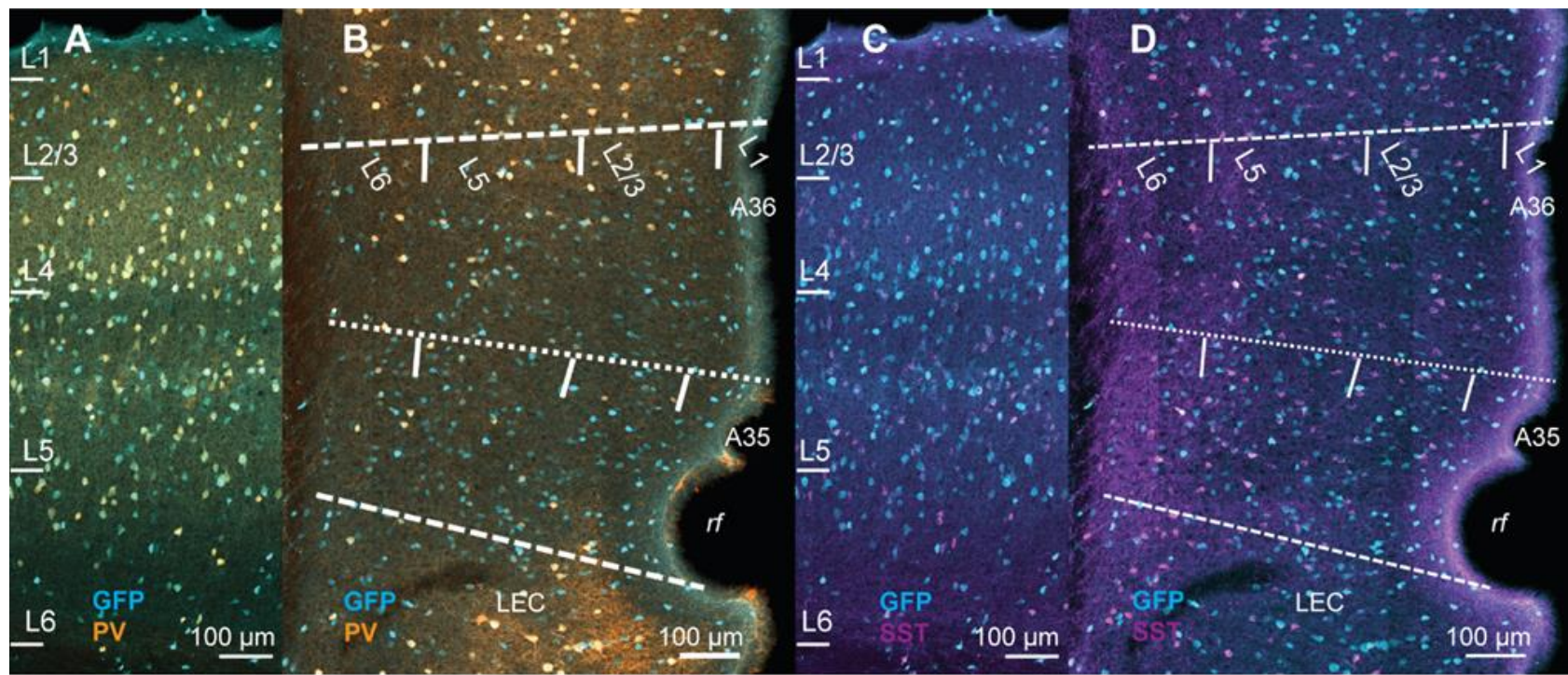

E

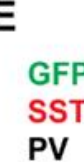

G

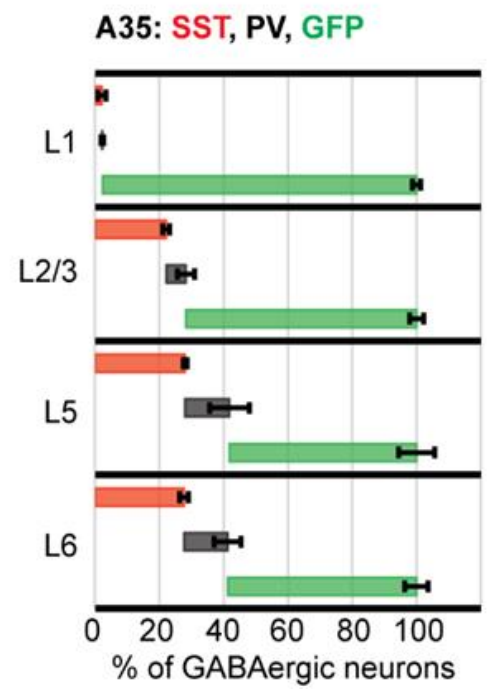

H

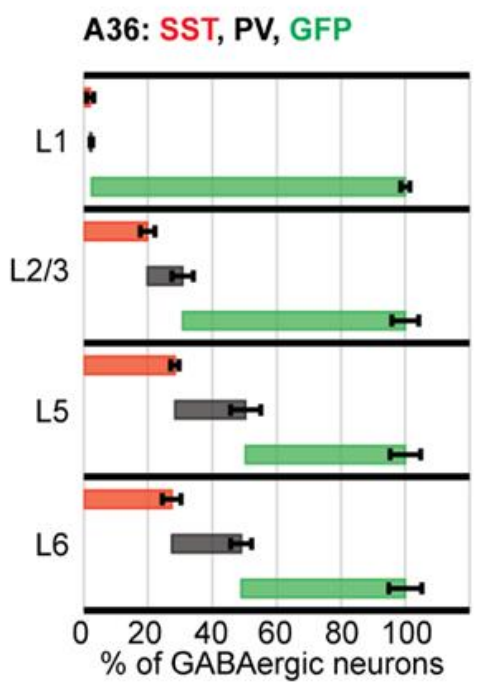

Page 2
$F$

A36

Total GABAergic population

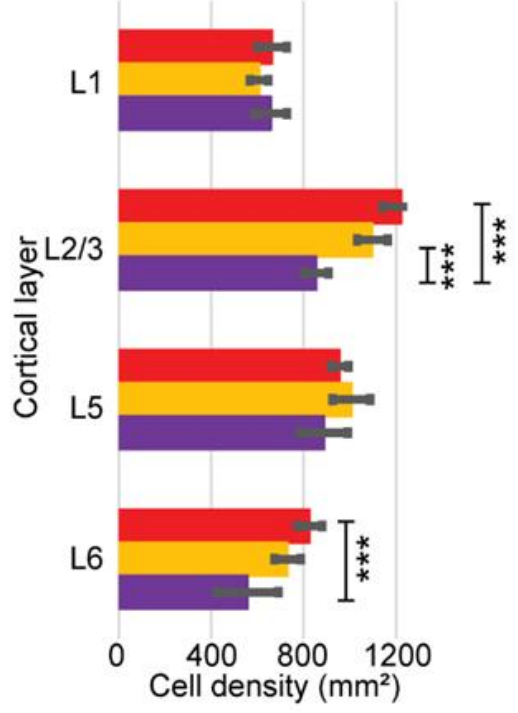

wS1: SST, PV, GFP

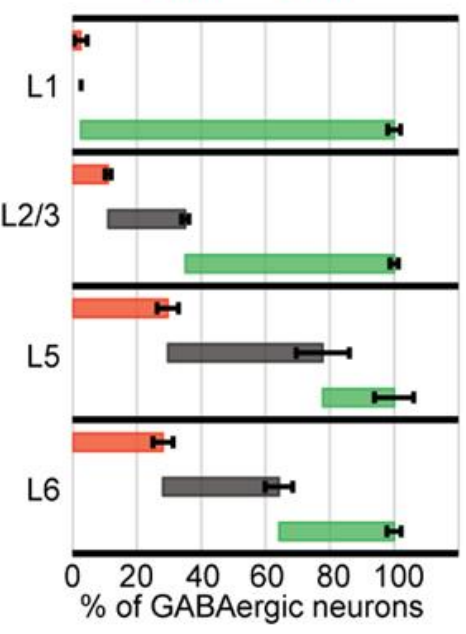




\begin{abstract}
Figure 1. A. Representative confocal stack of immunofluorescence for GFP (turquoise) and PV (orange) in the wS1 of a GAD67-GFP mouse. B. Representative confocal stack of immunofluorescence for GFP and PV in PER of a GAD67-GFP mouse. C. Representative confocal stack of immunofluorescence for GFP (turquoise) and SST (orange) in the wS1 of a GAD67-GFP mouse. D. Representative confocal stack of immunofluorescence for GFP and SST in PER of a GAD67GFP mouse. E. Graph plotting the samples in the principal component space using PC1 and PC2. F. Bar plot showing the statistical comparison of the density of GABAergic neurons across the layers of A35 (red), A36 (yellow) and wS1 (purple) ( $n=5$ mice): $L 1$ ( $F=1.33, d f=2, p=0.29$, One-Way ANOVA); $L 2 / 3(F=37.4, d f=2, p=6.9 E-6$, One-Way ANOVA; A35 vs wS1, $p=7.6 \mathrm{E}-6$, Bonferroni correction; $\mathrm{A} 36$ vs wS1, $p=0.0002$, Bonferroni correction); $L 5(F=2.8, d f=2, p=0.1$, One-way ANOVA); L6 ( $F=11.5, \mathrm{df}=2, \mathrm{p}=0.002$, One-Way ANOVA; A35 vs wS1, $p=0.008$, Bonferroni correction). G. Cumulative percentage of SST-IR (red), PV-IR (black) and GFP-IR/-PV-SST neurons in the total GABAergic population of A35. H. Cumulative percentage of SST-IR (red), PV-IR (black) and GFP-IR/PV-SST- neurons in the total GABAergic population of A36. I. Cumulative percentage of SST-IR (red), PV-IR (black) and GFP-IR/PV-SST- neurons in the total GABAergic population of wS1.
\end{abstract}

80 represents a gateway for sensory information 81 entering the hippocampal formation (Witter et 82 al., 2000), by excerting an inhibitory control on 83 the information travelling into the hippocampal 84 network (DeCurtis and Paré, 2004). The 85 mechanisms underlying this inhibitory control 86 are still poorly understood. One possibility is that 87 this inhibitory gating relies on strong perisomatic 88 feedforward inhibition mediated by PV-INs 89 (Willems et al 2018). This is in striking contrast 90 with the low density of PV-INs in PER. The low 91 density of PV-INs in PER suggests that either 92 the GABAergic population of PER is smaller 93 than that of the neocortex, or that other 94 GABAergic cell-types are enriched. In the 95 present study we aimed to explore these two 96 scenarios by combining mouse genetics, 97 molecular marker expression, enhancer 98 mediated transgene expression and 99 electrophysiological characterization of 100 GABAergic neurons in PER. We compared the 101 GABAergic population of PER to that of the area 102 of whisker representation of the primary 103 somatosensory cortex (wS1), whose 104 GABAergic diversity has been extensively 105 described (Lee et al., 2010; Feldmeyer et al., 1062018 ). We found that indeed the GABAergic 107 population is not smaller in PER and contains a 108 higher density of 5HT3aR-expressing 109 interneurons. Characterization of the firing 110 patterns of GABAergic neurons in PER shows 111 that this is partially due to an enrichment of late112 spiking neurogliaform cells. Most strikingly, we 113 also found that the percentage of fast-spiking 114 neurons was much higher than expected from 115 the density of PV-INs. Using an enhancer-driven 116 viral approach, we demonstrate that the 117 population of fast-spiking neurons in PER
118 includes PV-expressing neurons and a 119 population of neurons not expressing PV.

120

\section{Results}

122

123 Comparison of the GABAergic population of 124 wS1 and PER

125 To compare the whole GABAergic population of 126 PER and wS1 we used the GAD67-EGFP 127 mouse line and performed immunofluorescence 128 staining for PV, SST and GFP (Figure 1A-D). 129 We quantified the number of PV-IR, SST-IR and 130 GFP-IR/PV-SST- (neurons labeled by the 131 GAD67-EGFP mouse line but not expressing 132 either PV or SST) neurons across layers of the 133 two cortical areas and calculated their densities. 134 We performed a PCA on the densities of these 135 markers across the layers of wS1 and PER (A35 136 and A36) to test how these cell-types describe 137 these two cortical regions (Figure 1E). The first 138 two principal components accounted for $93.8 \%$ 139 of the variance and the data was well 140 segregated in the principal component space 141 (Figure 1E and Figure 1 supplement 2A). The 142 variables that best explained the variance were 143 the density of PV-INs in layers 2/3-6, which was 144 lower in PER, and the density of GFP-IR/PV145 SST- in layers 2/3-6, which was higher in PER 146 (Figure 1E and Figure 1 - figure supplement $1472 \mathrm{~B}$ ). The PCA results were confirmed by a 148 statistical comparison of the three molecularly 149 defined GABAergic populations (Figure 1 150 Figure supplement 3). Indeed, the density of PV151 INs was significantly lower in layer 2/3-6 of PER, 152 whereas the density of GFP/-PV-SST neurons 153 was significantly higher in L2/3-6 as compared 154 to wS1 (Figure 1 - Figure supplement 3 ). The 155 PCA analysis showed a slight contribution of 156 SST-INs in L2/3, and indeed their density was 


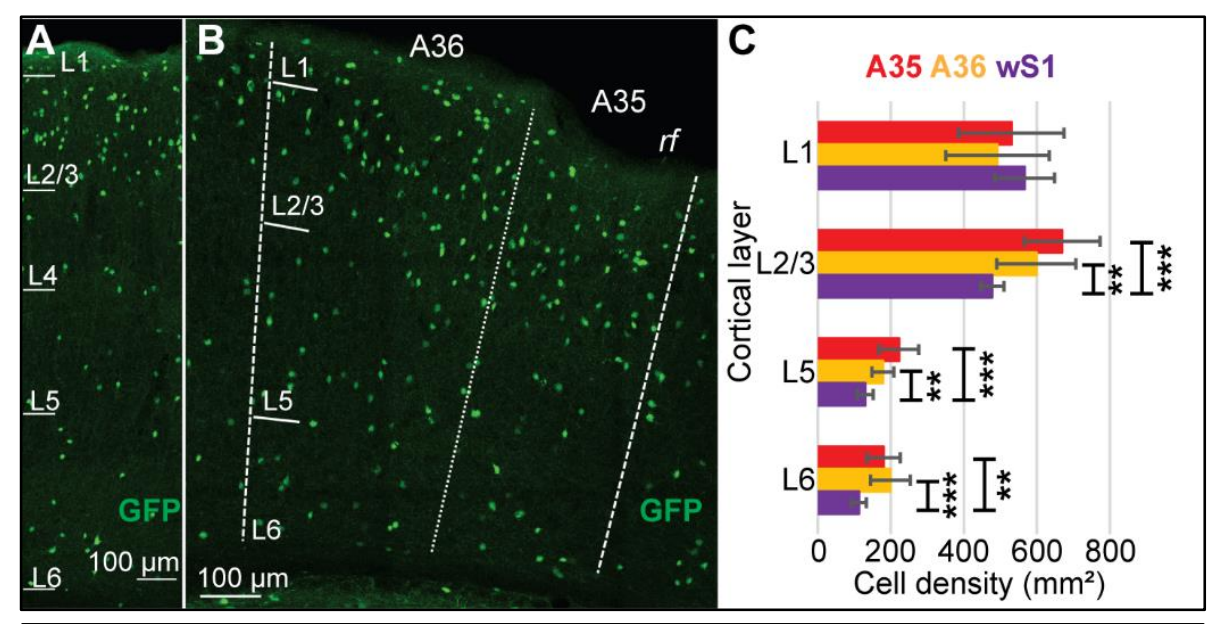

Figure 2. A. Representative confocal stack of immunofluorescence for GFP (green) in wS1 of the 5HT3aR-EGFP mouse line. B. Representative confocal stack of immunofluorescence for GFP (green) in PER of the 5HT3aR-EGFP mouse line. $\mathbf{C}$. Bar plot showing the statistical comparison of the density of GFP-IR neurons in the 5HT3aR-EGFP mouse line $(n=10$ slices in each area): $L 1(F=0.78, d f=2, p=0.47$, One-way ANOVA); L2/3 ( $F=10.81, d f=2, p=0.0004$, One-way ANOVA; A35 vs wS1, $p=0.0003$, Bonferroni correction; A36 vs wS1, $p=0.009$, Bonferroni correction); L5 $(F=13.24, d f=2, p=9.84 E-5$, One-way ANOVA; A35 vs wS1, $p=0.0005$, Bonferroni correction; A36 vs wS1, $p=0.001$, Bonferroni correction); L6 ( $F=10.02, d f=2, p=$ 0.0006, One-way ANOVA; A35 vs wS1, $p=0.002$, Bonferroni correction; A36 vs wS1, $p=0.0009$, Bonferroni correction).

157 significantly higher in L2/3 and 6 of PER (Figure $1581 \mathrm{E}$ and Figure 1 - Figure supplement 3). Our 159 data show that $A 35$ and $A 36$ are very similar in 160 their GABAergic population. However, the 161 densities of these GABAergic types in A36 are 162 between that of $\mathrm{A} 35$ and wS1 (Figure 1E and F, 163 Figure 1 - Figure supplement 3). These results 164 confirmed previous reports of low density of PV165 INs in PER (Whissel et al., 2015; Kim et al., 166 2017), and further show that PER is enriched in 167 a population of GABAergic neurons that does 168 not express either PV or SST.

169 Finally, we show that PER has a higher density 170 of GABAergic neurons, particularly in L2/3 and 171 L6 (Figure 1F). Our results suggest that in PER 172 the low density of PV-INs is compensated by an 173 enrichment of other classes of GABAergic 174 neurons. A comparison of the percentage of 175 marker-IR neurons in the GABAergic population 176 further confirmed the reduction of PV-INs and an 177 increased representation of GFP-IR/-PV-SST 178 neurons across the layers of $A 35$ and $A 36$ 179 (Figure 1G-I).

180

181 PER is enriched in GABAergic cell-types of 182 the 5HT3aR group

183 In sensory and motor areas of the dorsal cortex, 184 PV-INs represent the largest group of 185 GABAergic neurons (Tremblay et al., 2016). Our 186 results show that in PER the largest population
187 across layers does not express either PV or SST 188 (Figure 1). We reasoned that this population 189 might be in part composed of neurons 190 expressing GFP in the 5HT3aR-EGFP mouse 191 line, which do not express either PV or SST (Lee 192 et al., 2010). We tested this hypothesis by 193 quantifying the density of GFP labeled neurons 194 in the 5HT3aR-EGFP mouse line (Gong et al., 195 2003) (Figure 2). We found that PER contains a 196 significantly higher density of GFP labeled 197 neurons as compared to wS1, particularly in 198 L2/3, 5 and 6 (Figure 2C). We then tested 199 whether VIP-INs contributed to this difference 200 by performing immunolabelling for VIP in the 201 GAD67-EGFP mouse line (Figure 2 - Figure 202 supplement 1 ). The density of VIP-INs in PER 203 tended to be higher than in wS1, but the 204 difference was not significant, other than in L5 205 (Figure 2 - Figure supplement 1). We conclude 206 that the VIP-INs are not the major source of the 207 enrichment of 5HT3AR-INs in PER.

208

\section{L5 of PER is enriched of late-spiking 210 neurogliaform neurons}

211 The 5HT3AR-INs group includes VIP-INs, 212 Lamp5-INs (neurogliaform), and sncg-INs 213 (CCK-basket cells) (Tasic et al., 2018). Our 214 results show that VIP-INs provide only a small 215 contribution to the enrichment in 5HT3AR-INs in 216 PER (Figure 2 - Figure supplement 1). The 


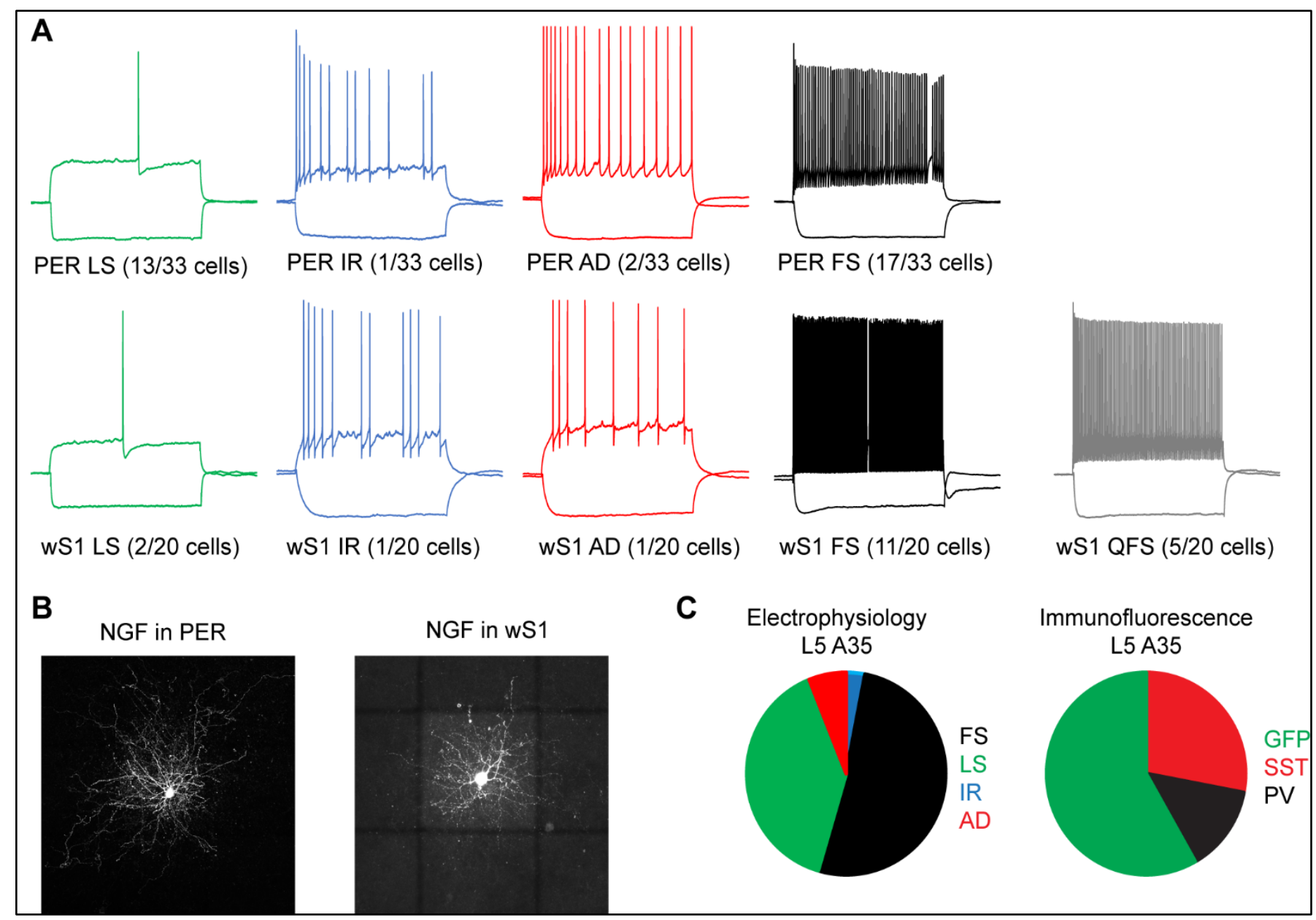

Figure 3. A. Firing patterns recorded from GABAergic neurons in L5 of A35 (upper row) and wS1 (lower row). B. Confocal stacks showing of a neurogliaform cell in PER (left) and wS1 (right). C. Comparison of the percentage of firing patterns (left) and molecularly defined populations (right) in L5 of A35. Note the high percentage of fast-spiking neurons as compared to the low percentage of PV-INs in PER.

217 other two groups lack specific mouse lines or 218 unique molecular markers. We used instead in 219 vitro electrophysiology to characterize the firing 220 patterns of GABAergic interneurons in L5 of 221 PER. We focused on L5 because it is where we 222 found the largest difference in the percentage of 223 GABAergic neurons not expressing PV and SST 224 between PER and wS1 (Figure 1G-I). 225 Neurogliaform cells in the cortex, hippocampus 226 and amygdala show a "late-spiking" phenotype 227 characterized by a long latency to the first spike, 228 broad AHPs and no adaptation (Overstreet229 Wadiche and McBain, 2015). Sncg-INs include 230 CCK-basket cells, and show a regular firing 231 pattern with little or no adaptation (Kawaguchi 232 and Kubota, 1998). We obtained brain slices 233 containing PER from GAD67-GFP mice and 234 performed whole-cell patch clamp experiments 235 in current clamp to measure voltage responses 236 of GABAergic neurons. We found four types of 237 firing patterns in PER ( $n=33$ cells): the fast-
238 spiking phenotype (51.5\%), associated to PV$239 \mathrm{INs}$, the irregular firing pattern (3.03\%), 240 associated to some VIP-INs, the late-spiking 241 phenotype (39.4\%), associated to neurogliaform 242 cells, and the adapting firing pattern (6.07\%), 243 associated to some SST-INs. In L5 of wS1 we 244 found five firing patterns ( $n=20$ cells): fast245 spiking (55\%), irregular (5\%), late-spiking $246(10 \%)$, adapting (5\%), quasi-fast spiking $(25 \%)$, 247 associated to layer 4 targeting non-Martinotti 248 SST-INs (Ma et al., 2006; Nigro et al., 2018) 249 (Figure 3A). Our electrophysiological 250 characterization of the GABAergic population in 251 PER and wS1 showed that late-spiking 252 neurogliaform cells are enriched in L5 of PER. 253 The late-spiking neurons in PER had short 254 dendrites and a dense local axonal arborization, 255 typical of neurogliaform cells in neocortical L1 256 and hippocampus (Overstreet-Wadiche and 257 McBain, 2015) (Figure 3B). We conclude that 258 neurogliaform cells might account for most of 


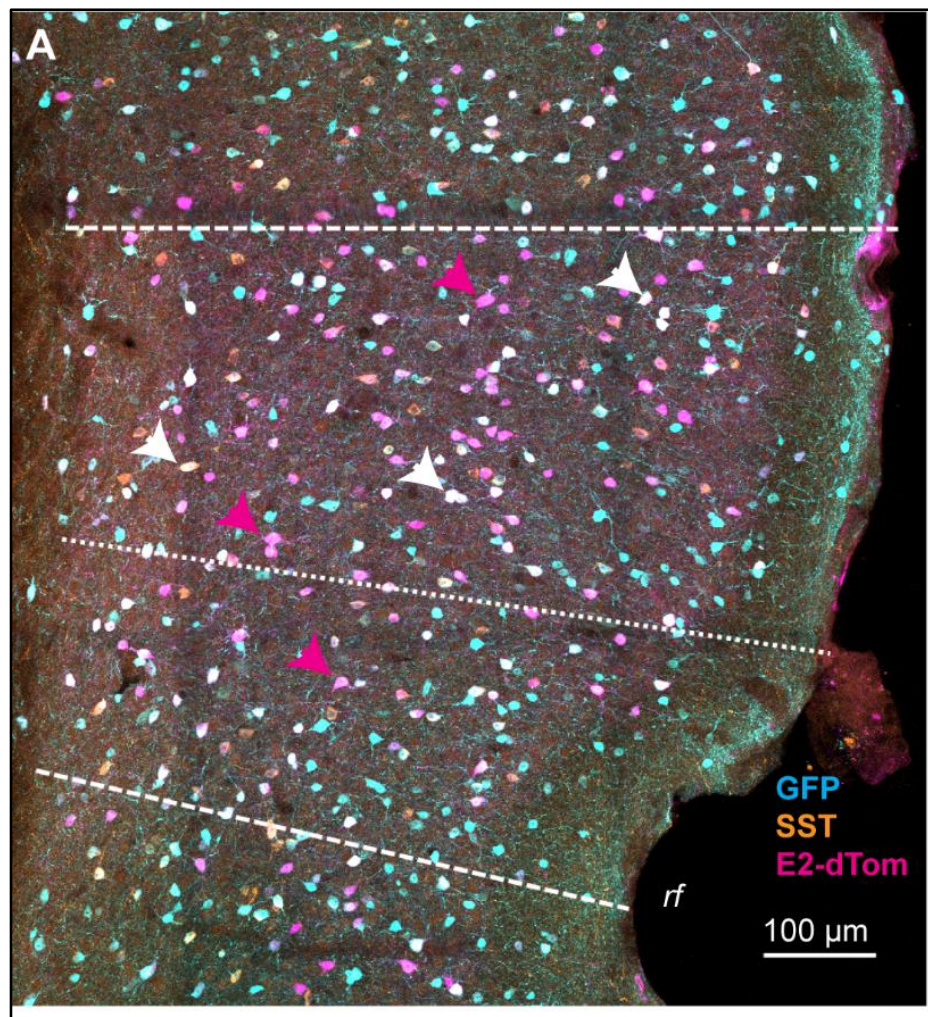

C

A35 L5 E2/SSTimmunofluorescence

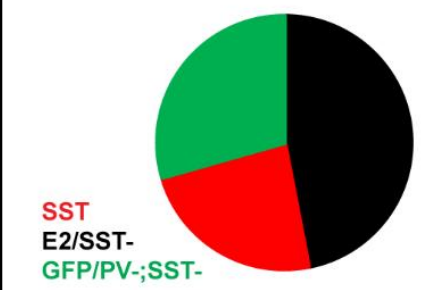

A35 L5 Firing patterns

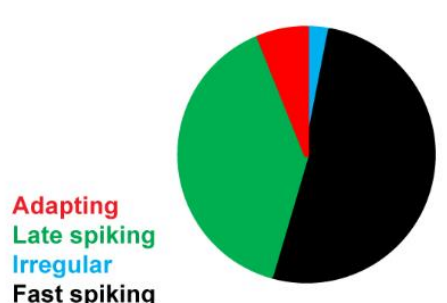

\section{B}

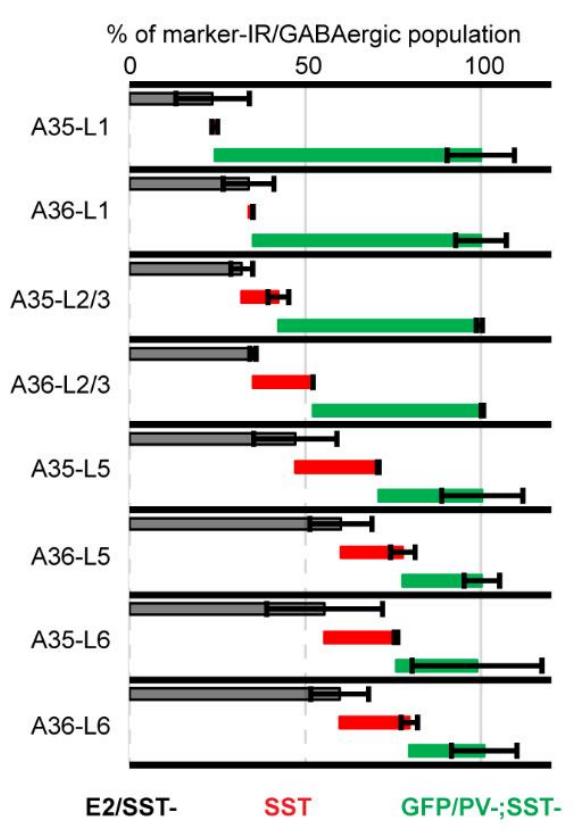

wS1 L5 PV-INs immunofluorescence

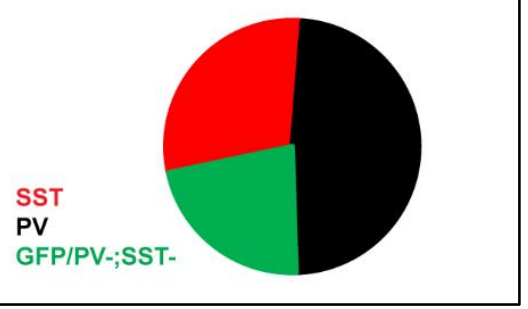

Figure 4. A. Representative confocal stack of immunofluorescence for GFP (turquoise) SST (orange) and dTomato (lilla) in PER of the GAD67-EGFP mouse line. B. Cumulative percentage of E2-dTom/SST- (black), SST-INs (red), GFP/PV-;SST- (green) neurons in the total GABAergic population of PER in the GAD67-EGFP mouse line ( $n=2$ mice). C. Pie charts comparing the percentage of marker defined populations in L5 A35 (left), electrophysiological characterization in L5 A35 (middle) and marker defined populations in L5 wS1. Note how the E2/SST- percentage is very similar to that of fast-spiking neurons in PER and PV-INs in wS1.

259 the difference in the density of 5HT3aR-INs 260 between PER and wS1.

261

262 A fast-spiking population that do not 263 express PV in PER

264 Unexpectedly, the fast-spiking phenotype 265 accounted for the largest population in PER and 266 wS1, which is at odds with the low density of PV267 INs in PER (Figure 1 and Figure 3C). We 268 hypothesized that some fast-spiking neurons in 269 PER do not express detectable levels of PV. To 270 test this hypothesis, we labelled fast-spiking 271 neurons with a viral approach that exploits 272 enhancer driven expression of tdTomato in PV273 INs throughout the brain (Vormstein-Schneider
274 et al., 2020). We injected the S5E2-dTom virus 275 in PER of GAD67-EGFP mice and stained for 276 SST to subtract the population of virus 277 transfected neurons that co-expressed SST 278 (Figure 4 - Figure supplement 1 and Figure 4 279 Figure supplement 2). We found that the E2$280 \mathrm{dTom} / \mathrm{SST}$ - represented the largest GABAergic 281 population in the deep layers of PER (Figure 4A$282 \mathrm{~B}$ ). The percentage of E2-dTom/SST- was 47.1 $283 \pm 11.8 \%$ of the total GABAergic population in L5 284 of $A 35$, which was very similar to the percentage 285 of PV-INs in L5 wS1 (48.3 $\pm 8.3 \%$ ) and to the 286 percentage of fast-spiking neurons in L5 of PER $287(51.5 \%)$ (Figure 4C). 


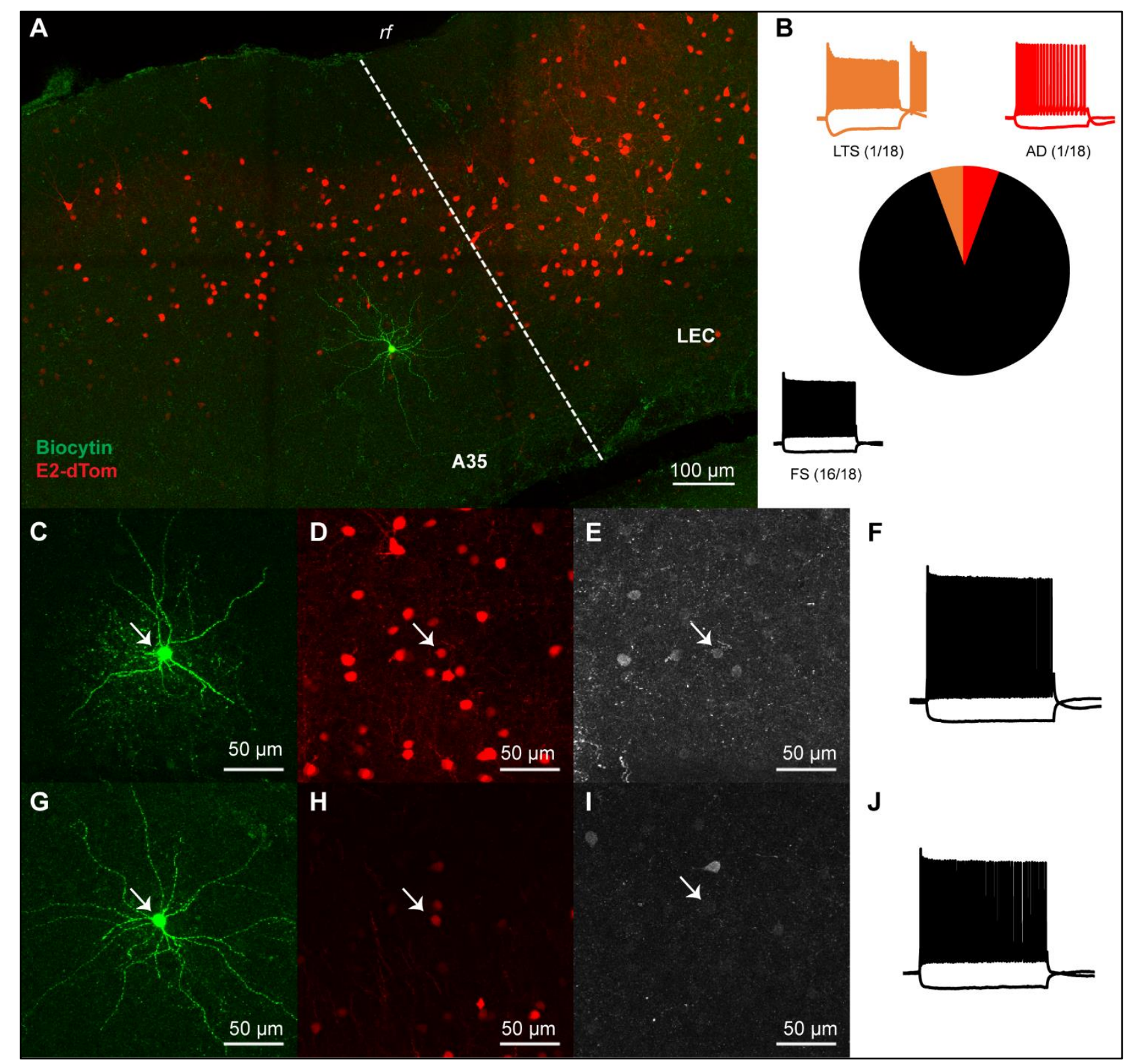

Figure 5. A. Confocal stack showing the location in L5 of A35 of a E2-dTom neuron filled with biocytin (green). Neurons labeled by the E2-dTom virus are shown in red. Dashed line shows the border with LEC. B. Pie chart showing the percent of LTS (orange), adapting (red) and fast-spiking (FS) (black) among E2-dTom neurons. C-E. Confocal stacks of a E2dTom neuron ( $D$, red) filled with biocytin (C, green) and expressing PV (E, white, white arrow). F. Voltage response of the cell shown in C-D to hyper- and depolarizing current steps. G-I. Confocal stacks of a E2-dTom neuron (H, red) filled with biocytin (G, green) and not expressing PV (I, white, white arrow). J. Voltage response of the cell shown in C-D to hyper- and depolarizing current steps.

288 This result further suggests that PER is 289 populated by a population of GABAergic 290 neurons expressing a fast-spiking phenotype 291 but not expressing PV. To directly test this 292 hypothesis, we performed in vitro patch clamp 293 recordings from brain slices of wild type C57 294 mice injected with the S5E2-dTom virus. We 295 found that $88.9 \%$ (16 out of 18 neurons) of 296 dTomato labelled neurons showed a fast-
297 spiking phenotype and $11.2 \%$ showed an 298 adapting (1/18) or low-threshold firing pattern 299 (1/18), typical of SST neurons (Figure 5A-B, and 300 Figure 5 - Figure supplement 1). We performed 301 immunofluorescence for PV on 15 of the 302 recorded fast-spiking neurons and found that 303 40\% (6/15) did not express PV (E2-PV-) (Figure $3045 \mathrm{C})$. 
bioRxiv preprint doi: https://doi.org/10.1101/2022.01.30.478360; this version posted February 8,2022 . The copyright holder for this preprint (which was not certified by peer review) is the author/funder, who has granted bioRxiv a license to display the preprint in perpetuity. It is made available under aCC-BY-NC-ND 4.0 International license.

305 Our results show that the fast-spiking population 306 of PER contains neurons that do not express 307 PV, highlighting a novel diversity within this 308 GABAergic type that is area specific.

\section{A}

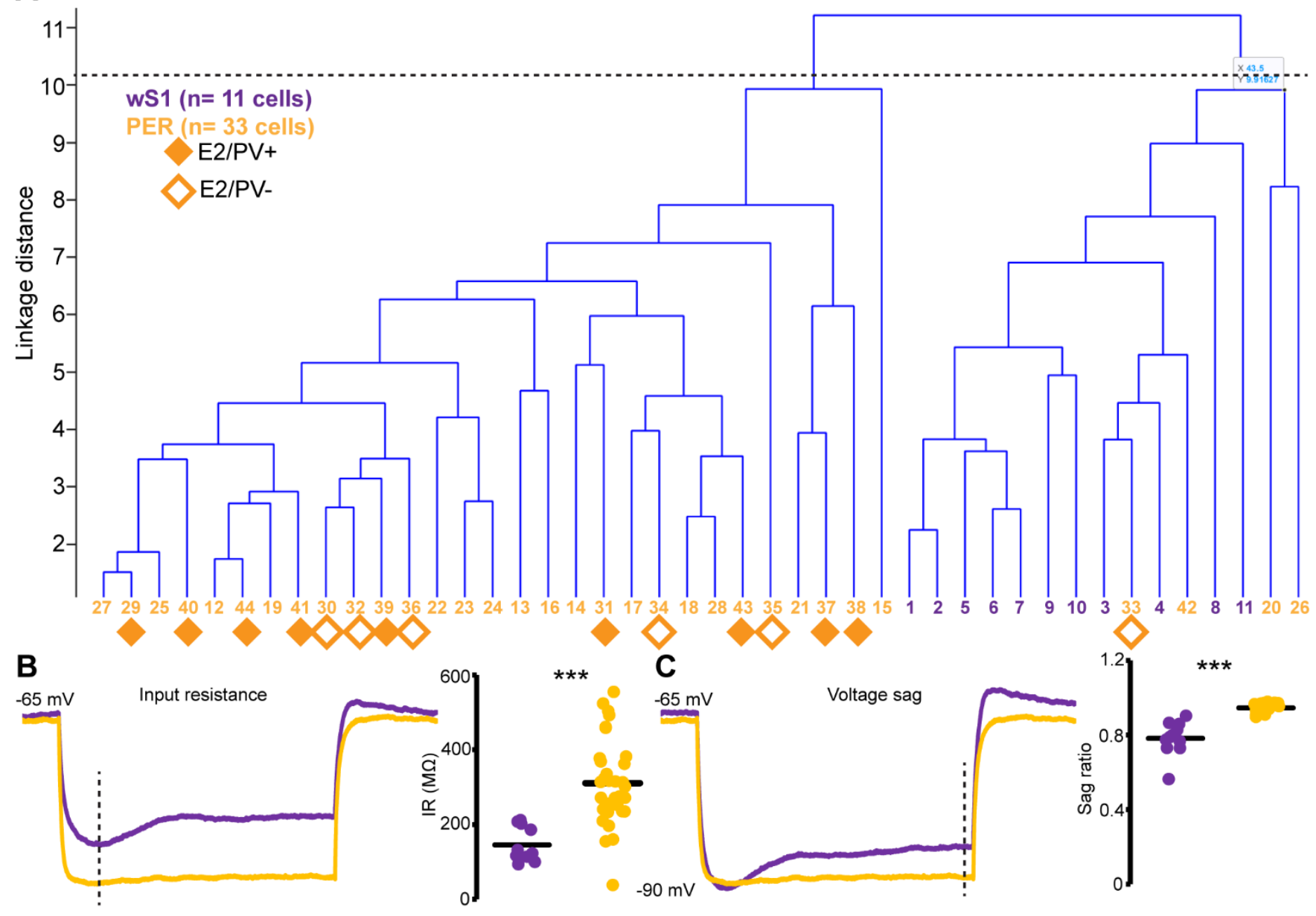

D

Action potential half width
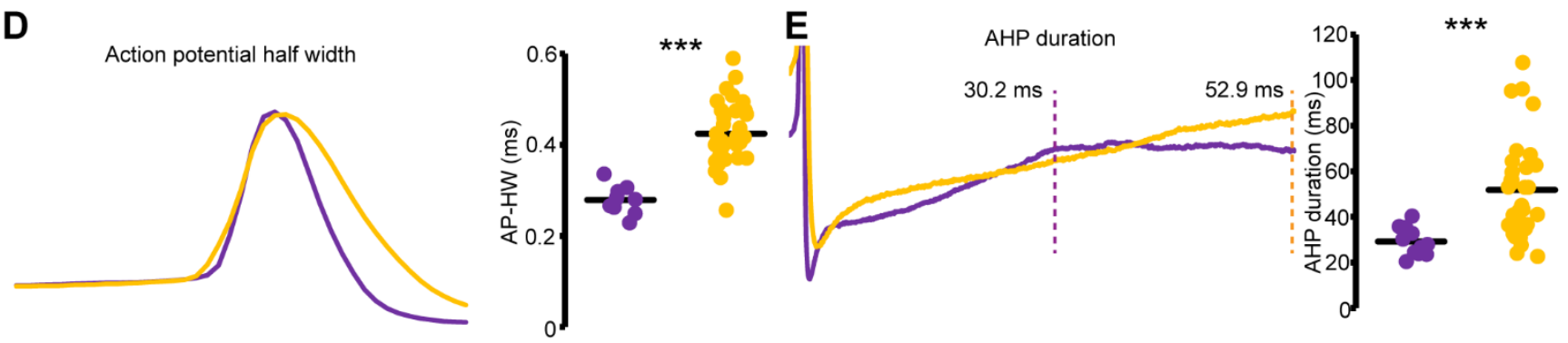

F

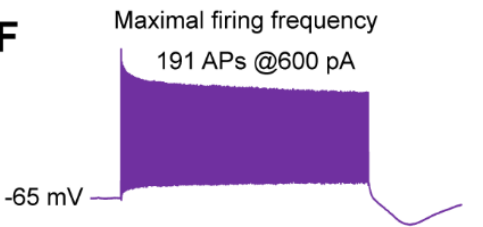

103 APs @250 pA

$-65 \mathrm{mV}$

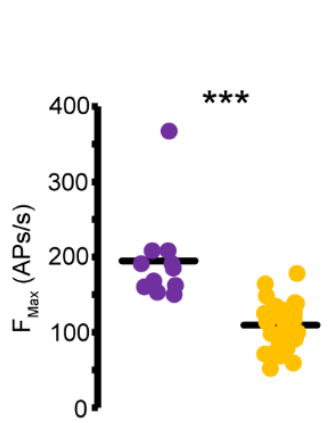

Input/output curve

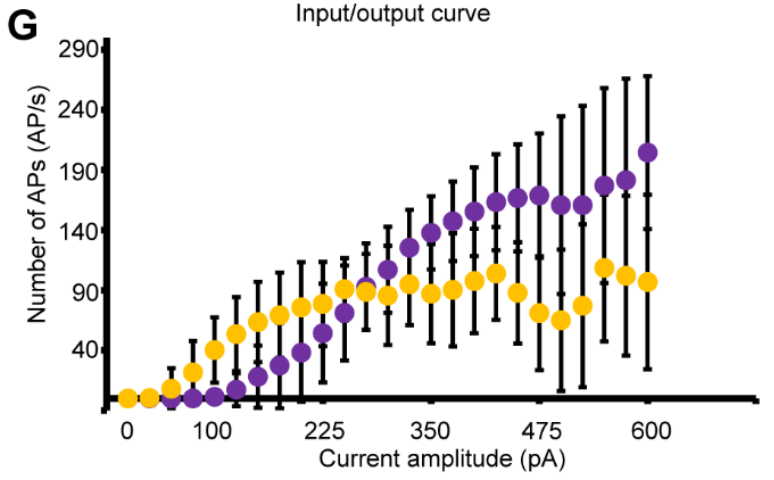

Page 8 
Figure 6. A. Dendrogram obtained from a hierarchical cluster analysis of 44 fast-spiking neurons (11 in wS1 and 33 in PER). The dashed line represents the level at which the clusters were identified by using the inconsistency index (see Methods). Numbers on the x-axis represent the cell's IDs color-coded according to the cortical area (yellow, PER; purple, wS1). Filled diamonds indicate E2-dTom neurons expressing PV. Open diamonds indicate E2-dTom neurons not expressing PV. B. Representative voltage response of fast-spiking neurons to a -100 pA current step in wS1 (purple) in PER (yellow) neurons. The dashed line indicates the peak of the response. The plot on the right shows the IR of all neurons (circles) and the population mean (black line) (wS1, $145.4 \pm 46.4 \mathrm{M} \Omega$; PER, $310.7 \pm 114.4 \mathrm{M} \Omega$; $\mathrm{p}=3.6 \mathrm{E}-8$, T-test). C. Representative voltage response to a hyperpolarizing current step of fast-spiking neurons in wS1 (purple) and in PER (yellow). All responses used to calculate the sag ratio reached $-90 \pm 2 \mathrm{mV}$. Note the difference in the steady-state response (dashed line). The plot on the right shows the sag ratio of all neurons (circles) and the population average (black line) (wS1, $0.78 \pm 0.1$; PER $0.94 \pm 0.02 ; p=0.0001$, T-test). D. Representative action potentials of fast-spiking neurons in wS1 (purple) and PER (yellow). The plot on the right shows the AP-HW of all neurons (circles) and the population mean (black line) (wS1, $0.28 \pm 0.03 \mathrm{~ms} ;$ PER, $0.42 \pm 0.07 \mathrm{~ms} ; \mathrm{p}=2.9 \mathrm{E}-12, \mathrm{~T}$-test). E. Representative AHPs following single APs in wS1 (purple) and PER (yellow). The AHPdur is indicated by the two dashed lines. The plot on the right shows the AHPdur of all neurons (circles) and the population mean (black line) (wS1, $29.1 \pm 6.2 \mathrm{~ms}$; PER, $51.8 \pm 21.3 \mathrm{~ms}$; $p=2.4 \mathrm{E}-6$, T-test). F. Representative responses of fast-spiking neurons in wS1 (purple) and PER (yellow) to depolarizing current steps eliciting maximal firing frequency. The plot on the right shows the Fmax in all neurons (circles) and the population mean (black line) (wS1, $194.8 \pm 60.7$ APs/s; PER, $109.5 \pm 29.6$ APs/s; $p=0.0008$, T-test). G. Plot of the average number of APs evoked with current steps of increasing amplitude in wS1 (purple) and PER (yellow).

309

310 Fast-spiking interneurons in PER show 311 different electrophysiological properties 312 than in wS1

313 To test whether the firing patterns of fast-spiking 314 neurons not-expressing PV in PER are different 315 from PV-INs in PER and wS1, we performed a 316 hierarchical cluster analysis on the 317 electrophysiological properties (Figure 6A). The 318 dendrogram shows that the population analyzed 319 can be divided in two clusters, one containing all 320 wS1 neurons, and a second containing all but 4 321 PER neurons (Figure 6A). The group containing 322 perirhinal fast-spiking neurons included PV-INs 323 and neurons not expressing PV, suggesting that 324 these neurons show similar electrophysiological 325 responses (Figure 6A). We further compared 326 the electrophysiological parameters included in 327 the cluster analysis (see Methods) and found 328 that fast-spiking interneurons in PER had a 329 significantly higher IR and as a consequence 330 they fired action potentials in response to 331 smaller current steps (Figure 6B and G). Fast332 spiking neurons in PER had a less pronounced 333 sag, which suggests a lower expression of $\mathrm{HCN}$ 334 channels (Figure 6C). A hallmark of PV-INs are 335 their fast APs and high firing rate, interestingly 336 fast-spiking neurons in PER had wider APs, 337 longer AHP and reached lower maximal firing 338 rates than their wS1 counterparts (Figure 6D$339 \mathrm{G})$. We did not find any difference between E2$340 \mathrm{PV}+$ and E2-PV- neurons in PER, suggesting 341 that these neurons might belong to the same 342 type and differ only in expression of PV.

343

\section{Discussion}

345 The present study offers a comprehensive 346 description of the GABAergic diversity in the 347 mouse PER, and demonstrates several 348 differences compared to a sensory region 349 (wS1). First, we demonstrate that PER contains 350 a comparable density of GABAergic neurons as 351 other cortical areas despite the low density of 352 PV-INs. Second, PER is enriched in 353 neurogliaform cells in layer 5 . Since our 354 molecular characterization showed no other 355 candidate for the increased density of 5HT3aR356 INs in other layers, we hypothesize that 357 neurogliaform cells are more numerous 358 throughout the layers of PER. Third, we 359 corroborated previous observations of an 360 increased SST-INs/PV-INs ratio in L2/3 (Kim et 361 al., 2017). We extend this observation by 362 showing that the density of SST-INs is 363 significantly higher in PER as compared to wS1. 364 Finally, we demonstrate that the fast-spiking 365 population of PER is comparable to that of wS1 366 and most of these neurons do not express PV. 367 We then show that the fast-spiking population of 368 PER shows several electrophysiological 369 differences with that in wS1 (and other 370 telencephalic areas), highlighting a novel 371 diversity within this electrophysiological cell372 type.

373

374 Enrichment of 5HT3aR-INs in PER

375 We found that, although the density of PV-INs is 376 lower in PER than in wS1, the GABAergic 377 population is of comparable size, or even larger 378 (e.g., in L2/3 and 6). This difference is partially 
379 due to an enrichment of GABAergic neurons 380 belonging to the neurogliaform type, and to a 381 lesser extent to the VIP-expressing group. 382 These GABAergic types belong to the CGE383 derived class (Tremblay et al., 2016). 384 Interestingly, transcriptomic analysis of the 385 human temporal cortex showed that the human 386 cortex is enriched of CGE-INs and 387 intratelencephalic projecting excitatory cells (IT) 388 (Hodge et al., 2019). CGE-INs and IT-projection 389 neurons show a preferential connectivity that is 390 established during development (Wester et al., 391 2019). The PER shows very sparse projections 392 outside of the telencephalon, except for the 393 thalamus, which suggests that a developmental 394 program might be responsible for the 395 enrichment of CGE-INs (McIntyre et al., 1996; 396 Benavidez et al., 2021).

397

398 Most fast-spiking GABAergic neurons in 399 PER do not express PV

400 The low expression of PV in PER has been used 401 by neuroanatomists to delineate PER for 402 several decades in different species (Pitcänen 403 and Amaral, 1993; Burwell et al., 1995; Uva et 404 al., 2004; Beaudin et al., 2013). However, this 405 observation has only recently been examined in 406 the context of GABAergic cell-types and 407 inhibitory circuits using specific mouse lines 408 targeting PV-INs (Whissel et al., 2015; Kim et 409 al., 2017). We have recently described that the 410 labelling in the PV-IRES-Cre mouse line is very 411 inefficient and quantifications of the number of 412 PV-INs would be dramatically underestimated 413 (Nigro et al., 2021). The present study 414 corroborates the low density of PV-INs in PER 415 by quantifying PV-IR neurons in PER and wS1. 416 However, our electrophysiological 417 characterization demonstrates that fast-spiking 418 interneurons represent half of the GABAergic 419 population in PER, similarly to wS1. Taking 420 advantage of a newly developed viral approach 421 we further demonstrate that most fast-spiking 422 neurons in PER do not express PV. The 423 electrophysiological properties of fast-spiking 424 interneurons in PER differ from those in wS1. 425 Our results suggest that the fast-spiking 426 population of the cortex shows a yet 427 unappreciated diversity. Recent transcriptomic 428 examination of the GABAergic cell-types of the 429 whole cortex did not identify transcriptomic 430 types with signatures of fast-spiking neurons but 431 not expressing PV (Yao et al., 2021). This is
432 likely due to the use of specific mouse lines 433 targeting different GABAergic types, which 434 would prevent the identification of a population 435 that do not express any of the main known 436 GABAergic molecular markers.

\section{7}

\section{Ideas and hypotheses}

439 The expression of PV is the hallmark of fast440 spiking GABAergic neurons throughout the 441 telencephalon (Yao et al., 2021). Our results 442 raise the hypothesis that $P V$ expression per se 443 might not be enough to define this GABAergic 444 class in all cortical areas. We have previously 445 shown that the PV-IRES-Cre mouse line shows 446 a low efficiency in several associative areas of 447 the cortex (Nigro et al., 2021). The low density 448 of PV-INs seems also to be a common feature 449 of associative areas (Whissel et al., 2015; Kim 450 et al., 2017). We hypothesize that our results 451 might extend beyond PER to other associative 452 areas of the cortex. Transcriptomic analysis of 453 neurons labeled by the S5E2-dTom virus might 454 reveal the taxonomic relationship between 455 these different types of fast-spiking neurons in 456 the cortex.

457 Until now we have assumed that fast-spiking 458 neurons not expressing PV belong to the PV 459 GABAergic class based on our 460 electrophysiological characterization. The lack 461 of PV expression might be due to plasticity of the 462 circuit in which these cells are embedded. 463 Indeed, PV expression is activity dependent and 464 has been shown to be modulated by learning 465 (Donato et al., 2013).

466 An alternative scenario could be that these 467 neurons belong to a different class (e.g., Lamp5, 468 scng, SST, or VIP), and simply lost their 469 characteristic marker. Interestingly, the S5E2470 dTom virus labels all PV-INs and about $60 \%$ of 471 the SST population. The fast-spiking phenotype 472 described here in perirhinal neurons is 473 reminiscent of the quasi-fast spiking phenotype 474 of non-Martinotti neurons described in L4 and 475 L5b of wS1 (Ma et al., 2006; Nigro et al. 2018; 476 Naka et al. 2019). However, our 477 characterization of the firing pattern of SST 478 neurons in L5 of PER shows that these neurons 479 express either an adapting or a low-threshold 480 spiking phenotype. Moreover, non-Martinotti 481 neurons are extremely rare outside of wS1 (Ma 482 et al., 2006; Scala et al., 2019). Finally, some 483 chandelier cells do not express PV, and express 484 a firing pattern similar to that described here in 
485 PER (Taniguchi et al., 2013). For these reasons 486 we believe that the fast-spiking population in 487 PER belongs to the PV transcriptomic class but 488 lacks PV expression.

\section{9}

\section{Methods}

491 Animal models

492 The research described here was performed on 493 adult mice (>8 weeks old) of both sexes. We 494 used the following mouse lines: C57BL/6J line 495 from Jackson Laboratories (stock 000664), 496 GAD67-eGFP obtained as gift from Dr. 497 Yanagawa (Tamamaki et al., 2003), the PV498 IRES-Cre line (B6;129P2-Pvalbtm1(cre)arbr/J, 499 stock 008069); R26R-EYFP (129X1$500 \mathrm{Gt}(\mathrm{ROSA}) 26 \mathrm{Sortm} 1$ (EYFP)Cos/J stock $501006148)$ and the SST-Cre line 502 (Ssttm2.1(cre)Zjh/J, stock 013044). We used 503 two brains of the 5HT3aR-EGFP mouse line 504 (MMRRC: $\quad \mathrm{Tg}$ (Htr3a-EGFP)DH30Gsat/Mmnc 505 stock: 000273-UNC) kindly donated by Dr. 506 Ramesh Chittajallu. The PV-IRES-Cre line was 507 crossed with the R26R-EYFP to obtain EYFP 508 expression in PV-INs. The PV-IRES-Cre was 509 bred as homozygous, whereas the GAD67510 eGFP and the R26R-EYFP were bred as 511 heterozygous. Animals were bred in house, 512 group housed in enriched cages with water and 513 food ad libitum. Animals were kept with an 514 inverted dark/light cycle of $12 \mathrm{~h} / 12 \mathrm{~h}$.

\section{5}

516 AAV2/1-S5E2-dTomato

517 purification

518 The plasmid construct for the S5E2-dTom was 519 purchased from Addgene (\#135630) 520 (Vormstein-Schneider et al., 2020). We 521 packaged the construct in AAV 2/1 serotype (a 522 mosaic of capsid 1 and 2) and generated 523 injection-grade viral vector using Heparin 524 column affinity purification method (Nair et al 525 iScience 2020). All the plasmids for AAV vector 526 preparations were made using endotoxin free 527 plasmid maxiprep kit (\#12663, Qiagen). AAV 528293 cells (\#CVCL_6871, Agilent, USA) used for 529 vector productions were maintained in DMEM (\# 53041965062 , Thermo Fisher Scientific) containing $53110 \%$ fetal bovine serum (\#16000-044, 532 ThermoFisher, USA) and penicillin/streptomycin 533 antibiotics (\#15140122, Thermo Fisher 534 Scientific). The day before transfection, we 535 seeded approximately 7x 106 AAV 293 cells on $536150 \mathrm{~mm}$ cell culture plates and maintained at $53737^{\circ} \mathrm{C}, 5 \% \mathrm{CO} 2$. For the calcium chloride
538 mediated co-transfection we used $22.5 \mu \mathrm{g}$ 539 pAAV-containing the transgene, 22.5 $\mu \mathrm{g}$ 540 pHelper (\#240071, Agilent, USA), $11.3 \mu \mathrm{g}$ pRC 541 (\#240071, Agilent, USA) and $11.3 \mu \mathrm{g}$ pXR1 542 (NGVB, IU, USA) capsid plasmids. The culture 543 medium was replaced after 7 hours with fresh 544 10\% FBS containing DMEM. Transfected cells 545 were scrapped out after 72 hours and 546 centrifuged at $200 \mathrm{xg}$. The pellet was subjected 547 to lysis using a buffer containing $150 \mathrm{mM} \mathrm{NaCl}$ $54820 \mathrm{mM}$ Tris $\mathrm{pH} 8.0$ and $10 \%$ sodium deoxy 549 cholate. The lysate was then treated with 550 Benzonase nuclease HC (\#71206-3, Millipore) 551 for 45 minutes at 37 oC. Subsequently, the 552 benzonase treated lysate was centrifuged at $5533000 x g$ for 15 mins. The clear supernatant was 554 then subjected to HiTrap® Heparin High 555 Performance (\#17-0406-01, GE) affinity column 556 chromatography using a peristaltic pump. The 557 elute from the Heparin column was then 558 concentrated using Amicon Ultra centrifugal 559 filters (\#Z648043, Millipore). We performed 560 quantitative PCR on the viral stock and the titer 561 was determined as approximately 1011 562 infectious particles $/ \mathrm{ml}$.

563

564 Injections of S5E2-dTomato viral vector

565 The AAVs were injected into the brains of C57 $566(n=6)$, GAD67-EGFP $(n=2)$ and on PV-EYFP $567(n=2)$ mice. Mice were anaesthetized with 568 isoflurane (4\% Nycomed, airflow $1 \mathrm{l} / \mathrm{min}$ ) in an 569 induction chamber. The mice were placed in a 570 stereotactic frame on a heated pad $\left(37^{\circ} \mathrm{C}\right)$ 571 throughout the procedure and head-fixed with 572 ear bars. Eye ointment was applied to protect 573 the cornea. The following analgesic were 574 applied subcutaneously: buprenorphine 575 hydrochloride $(0.1 \mathrm{mg} / \mathrm{Kg}$, Temgesic, Invidior), 576 meloxicam $1 \mathrm{mg} / \mathrm{Kg}$, Metacam Boerringer 577 Ingelheim Vetmedica), bupivacaine 578 hydrochloride $(1 \mathrm{mg} / \mathrm{Kg}$ locally, Marcain, Astra 579 Zeneca). The skin on the incision site was 580 shaved and disinfected with Pyrisept. An 581 incision was made to access the skull. The skull 582 was thinned with a dental drill at the desired 583 location, and a hole was punched with the tip of 584 a glass pipette. We used the following 585 coordinates from bregma: for wS1 $-0.7 \mathrm{~mm}$ AP, $586+3.1 \mathrm{~mm} \mathrm{ML},-0.15$ and $0.55 \mathrm{DV}$; for PER $-3 \mathrm{~mm}$ $587 \mathrm{AP},-1.5 \mathrm{DV}$, for the ML the pipette was moved 588 to the edge of the skull and then retracted 0.4 $589 \mathrm{~mm}$. Injections of $100-200 \mathrm{nl}(50 \mathrm{nl} /$ minute) in 590 each location were made with a glass pipette 
591 (20-30 $\mu \mathrm{m}$ tip size) attached to an injector 592 (Nanoliter 2010, World Precision Instruments) 593 controlled by a microsyringe pump controller 594 (Micro4 pump, World Precision Instruments). 595 After retracting the pipette, the wound was 596 rinsed with saline and sutured. The mice were 597 allowed to recover in a heated chamber $\left(37^{\circ} \mathrm{C}\right)$ 598 before returning to the homecage. 599 Postoperative analgesic was applied 6-8 $\mathrm{h}$ after 600 the procedure (Temgesic) and $24 \mathrm{~h}$ after the 601 procedure (Metacam). The survival time for 602 transduction and expression of the genetic 603 material was $10-15$ days.

\section{4}

605 In vitro electrophysiology

606 Mice of either sex (>8-week-old) were 607 euthanized with an overdose of pentobarbital 608 (i.p. $100 \mathrm{mg} / \mathrm{Kg}$, Apotekerforeninger), before 609 intracardial perfusion with cutting solution (RT) 610 of the following composition (in $\mathrm{mM}$ ): 93 choline 611 chloride, $3 \mathrm{KCl}, 1.25 \mathrm{NaH} 2 \mathrm{PO} 4,30 \mathrm{NaHCO} 3,20$ 612 HEPES, 10 glucose, $5 \mathrm{MgCl} 2,0.5 \mathrm{CaCl} 2,5 \mathrm{~N}$ 613 acetylcysteine, saturated with $95 \%$ O2 and 5\% $614 \mathrm{CO} 2$. The brain was extracted and a section 615 containing either PER or wS1 was glued onto 616 the stage of a vibratome (VT1000, Leica) filled 617 with cold $\left(4^{\circ} \mathrm{C}\right)$ cutting solution. Slices $(300 \mu \mathrm{m})$ 618 were transferred to a chamber filled with warm $619\left(34^{\circ} \mathrm{C}\right)$ cutting solution and incubated for 15 620 minutes. The slices were stored in a different 621 chamber filled with the following solution (in $\mathrm{mM}$ ) 622 (RT): $92 \mathrm{NaCl}, 3 \mathrm{KCl}, 1.25 \mathrm{NaH} 2 \mathrm{PO} 4,30$ $623 \mathrm{NaHCO}$, 20 HEPES, 10 glucose, $5 \mathrm{MgCl}$, 0.5 $624 \mathrm{CaCl}$, $5 \mathrm{~N}$-acetylcysteine, saturated with 95\% $625 \mathrm{O} 2$ and $5 \% \mathrm{CO} 2$.

626 The slices were placed in a recording chamber 627 of an upright microscope (BW51, Olympus) 628 filled with warm $\left(35^{\circ} \mathrm{C}\right)$ recording solution of the 629 following composition: $124 \mathrm{NaCl}, 3 \mathrm{KCl}, 1.25$ $630 \mathrm{NaH} 2 \mathrm{PO} 4,26 \mathrm{NaHCO} 3,10$ glucose, $1 \mathrm{MgCl}$, $6311.6 \mathrm{CaCl} 2$, saturated with $95 \% \mathrm{O} 2$ and $5 \% \mathrm{CO} 2$. 632 Characterization of the electrophysiological 633 properties was performed in presence of 634 synaptic blockers: $10 \mu \mathrm{M}$ DNQXNa2, $25 \mu \mathrm{M}$ $635 \mathrm{APV}$ and $10 \mu \mathrm{M}$ gabazine. wS1 was identified 636 under DIC optics by the presence of 637 characteristic barrels in layer 4. To identify A35 638 under DIC optics, we first identified the lateral 639 entorhinal cortex (LEC) by its characteristic 640 large neurons in layer $2 a$, and by the clear 641 separation between layer $2 a$ and $2 b$. A35 was 642 defined as the cortex dorsally located to LEC. 643 Only healthy, fluorophore-expressing neurons
644 were selected for recordings and no other 645 criterion was applied. Whole-cell patch clamp 646 recordings were performed with borosilicate 647 pipettes (Sutter Instruments) with a resistance 648 of 3-7 M $\Omega$ filled with the following solution (in $649 \mathrm{mM}$ ): $130 \mathrm{~K}$-gluconate, $10 \mathrm{KCl}, 10$ HEPES, 0.2 650 EGTA, 4 ATP-Mg, 0.3 GTP-Na, 5 651 phosphocreatine-Na2, $\mathrm{pH}$ 7.3. In all recordings 652 biocytin was added to the pipette solution to 653 recover the layer location of the neuron. 654 Membrane potentials reported were not 655 corrected for a junction potential of $-14 \mathrm{mV}$. 656 Current clamp recordings were performed with 657 a Multiclamp 700B amplifier (Molecular 658 Devices), digitized with a 1550A Digidata 659 (Molecular Devices), interfaced with a personal 660 computer with pClamp 11 (Molecular Devices). 661 Data were sampled at $40 \mathrm{kHz}$ and low-passed 662 filtered at $10 \mathrm{kHz}$. All recordings were performed 663 from a holding potential of $-65 \mathrm{mV}$. The 664 electrophysiological parameters analyzed were 665 defined as follows:

666 Input resistance (IR, M $\Omega$ ): resistance measured 667 from Ohm's law from the peak of voltage 668 responses to $-25 \mathrm{pA}$ hyperpolarizing current 669 steps.

670 Sag ratio (dimensionless): measured from 671 voltage responses peaking at $-90 \pm 2 \mathrm{mV}$ to 672 hyperpolarizing current steps. It is defined as the 673 ratio between the voltage at the steady-state 674 response and the peak.

675 Action potential threshold (APthr, mV): 676 measured from action potentials (AP) evoked 677 near rheobase, and defined as the voltage 678 where the rise of the AP was $20 \mathrm{mV} / \mathrm{ms}$.

679 AP half width (HW, ms): duration of the AP at 680 half amplitude from APthr, measured from 681 action potentials (AP) evoked near rheobase.

682 Amplitude of the fast afterhyperpolarization 683 (fAHP, $m V$ ): measured from APthr, from action 684 potentials (AP) evoked near rheobase.

685 AHP duration (AHPdur, ms): measure from 686 action potentials (AP) evoked near rheobase, 687 and defined as the time difference between the $688 \mathrm{fAHP}$ and the most depolarized voltage in a 200 $689 \mathrm{~ms}$ window.

690 Maximal firing frequency (Fmax, AP/s): maximal 691 firing frequency evoked with 1-s-long 692 depolarizing current steps.

693 Rheobase (Rheo, pA): current level that evoked 694 the first spike during a 1-s-long depolarizing $695 \mathrm{ramp}(500 \mathrm{pA} / \mathrm{s})$.

696 


\section{Histology}

698 Mice used for histological analysis were 699 anaesthetized with isoflurane and then 700 euthanized with an overdose of pentobarbital 701 (i.p. $100 \mathrm{mg} / \mathrm{Kg}$, Apotekerforeninger). The mice 702 were intracardially perfused with Ringer solution $703(0.85 \% \mathrm{NaCl}, 0.025 \% \mathrm{KCl}, 0.02 \% \mathrm{NaHCO} 3)$ 704 followed by $4 \%$ parafolmaldehyde (PFA) in 705 phosphate buffer (PB) (pH 7.4). The brain was 706 extracted from the skull and placed in 4\% PFA 707 for 3 hours before moving it to a solution 708 containing $15 \%$ sucrose in PB overnight. The 709 brains were then stored in $30 \%$ sucrose for two 710 days before being sliced at $50 \mu \mathrm{m}$ with a 711 freezing microtome. Slices were collected in six 712 equally spaced series and placed in tubes 713 containing a solution composed of $30 \%$ glycerol, $71430 \%$ ethylene glycol and $40 \%$ phosphate buffer 715 saline (PBS). The tissue was stored at $-20{ }^{\circ} \mathrm{C}$ 716 until used for histology.

717 The slices were washed in PB ( $3 \times 10$ minutes) 718 before incubation with blocking solution $(0.1 \%$ 719 Triton-X, 10\% NGS in PB). After blocking the 720 tissue was incubated with the primary antibody 721 for three days at $4{ }^{\circ} \mathrm{C}$ in a solution containing: $7220.1 \%$ Triton-X, 1\% NGS in PB. After being 723 washed ( $3 \times 1$ hour in $P B$ ), the tissue was 724 incubated with the secondary antibody 725 overnight at $4{ }^{\circ} \mathrm{C}$. The slices were washed $(3 \mathrm{X}$ 72610 minutes in PB) and mounted on SuperFrost 727 slides (Termo Fisher Scientific) and left to dry 728 overnight. The slides were coversliped with 729 entellan xylene (Merck Chemicals, Darmstadt, 730 Germany) after being washed in xylene for 15 731 minutes, or with Fluoromount after being 732 washed in distilled water for about 30 seconds. $733 \mathrm{We}$ used the following primary antibodies: 734 Guinea Pig IgG anti-NeuN (1:1000, Sigma 735 Millipore, \#ABN90P), Rabbit anti-parvalbumin 736 (1:1000, Swant, \#PV-27), Mouse IgG1 anti737 parvalbumin (1:1000, Sigma, \#P3088), Rat 738 lgG2a anti-RFP (1:1000, Proteintech, \#5f8), 739 Chicken IgY anti-GFP (1:1000, Abcam, 740 \#ab13970), Rabbit IgG anti-SST (1:1000, BMA 741 Biomedicals), Rabbit IgG anti-VIP (1:1000, 742 Immunostar). We used the following secondary 743 antibodies: Goat anti-guinea pig (IgG H+L) A647 744 (1:500, Invitrogen, \#A-21450), Goat anti-Rat 745 (IgG H+L) A-546 (1:500, Invitrogen, \#A11081), 746 Goat anti-Rabbit (IgG $\mathrm{H}+\mathrm{L})$ A488 (1:500, 747 Invitrogen, \#A11008), Goat anti-chicken (IgY $748 \mathrm{H}+\mathrm{L})$ A488 (1:500, Invitrogen, \#A11039), Goat 749 anti-rabbit (IgG $\mathrm{H}+\mathrm{L})$ A546 (1:500, Invitrogen,
750 \#A11010), Goat anti-rabbit (lgG $\mathrm{H}+\mathrm{L}) \quad$ A635 751 (1:500, Invitrogen, A31576).

752

753 Image acquisition and analysis

754 We used a confocal microscope (Zeiss LSM 880 755 Axiolmager Z2) to image regions of interest 756 (ROls) to count labeled neurons. Images of PER 757 and wS1 were taken with a x20 air objective with 7581 Airy unit pinhole size and saved as czi files. 759 We selected 3 to 5 ROls for each cortical region. 760 ROls contained the whole cortical area of 761 interest in a given slice. Files containing the 762 ROls were uploaded in Neurolucida (Micro 763 Bright Field Bioscience) for analysis. We 764 created contours to delineate the layers of the 765 ROls using the NeuN signal. The PER was 766 divided in area 35 (A35) and area 36 (A36), and 767 delineated according to Beaudin et al. (2013). 768 Symbols for each signal were used to count 769 cells labeled by different markers. We counted 770 all cells contained within the contours. Since we 771 counted slices distanced $300 \mu \mathrm{m}$ from each 772 other, overcounting in the $z$ axis is not relevant 773 and we did not apply any correction. 774 Quantification of the number of markers in each 775 contour was done in Neurolucida Explorer 776 (Micro Bright Field Bioscience) and exported in 777 excel files. The density of neurons labeled by a 778 marker was measured as the number of labeled 779 neurons in a contour divided by the area of the 780 contour (cells/mm2). The GAD67-eGFP mouse 781 line labelled virtually all PV-INs and VIP-INs, but 782 showed a lower specificity for SST-INs (Figure 7831 - Figure supplement 1). For this reason, the 784 number of PV-INs, SST-INs and VIP-INs was 785 measured from the immunolabelling for the 786 specific marker, independently of GFP 787 expression. Transcriptomic analysis of the 788 neuronal population of the cortex shows that 789 SST, VIP and PV are expressed exclusively in 790 GABAergic neurons. We excluded from the 791 counting a small population of PV-IR pyramidal 792 neurons in L5B of wS1 (van Brederode et al., 793 1991). Values are reported as mean \pm standard 794 deviation). All graphs were created in excel, 795 images of ROls were created in Zen lite (Zeiss) 796 and figures in illustrator.

797

798 Statistics

799 Principal component analysis on the densities of 800 different GABAergic neurons across layers of 801 PER and wS1 was performed in Matlab 2021 802 (Mathworks) using the function "pca". We 
803 obtained the explained variance for each 804 principal component, the principal component 805 coefficients, the principal component scores, 806 and the principal component variances. 807 Hierarchical cluster analysis was performed in 808 Matlab 2021 (Mathworks). After normalization 809 with the function "zscore", we calculated the 810 distance between pairs of objects with the 811 function "pdist" and the method "cityblock". 812 Objects were grouped according to the 813 calculated distances with the function "linkage" 814 and the method "average". After calculating the 815 inconsistency index ("inconsistent"), clusters 816 were obtained by using the function "cluster" 817 and the inconsistency index as cutoff. This 818 method identified two clusters.

834

835

836

\section{Acknowledgments}

838 We thank Dr. Ramesh Chittajallu for the kind donation of the brains of the 5HT3aR-EGFP mice and Dr. 839 Timothy Petros for helping with obtaining the brain of the 5HT3aR-EGFP mice.

\section{0}

\section{References}

842

843 Beaudin, S. A., Singh, T., Agster, K. L., \& Burwell, R. D. (2013). Borders and comparative 844 cytoarchitecture of the perirhinal and postrhinal cortices in an F1 hybrid mouse. Cerebral cortex, 23(2), 845 460-476.

846 Benavidez, N. L., Bienkowski, M. S., Zhu, M., Garcia, L. H., Fayzullina, M., Gao, L., . . Cotter, K. R. 847 (2021). Organization of the inputs and outputs of the mouse superior colliculus. Nature Communications, 848 12(1), 1-20.

849 Burwell, R. D., Witter, M. P., \& Amaral, D. G. (1995). Perirhinal and postrhinal cortices of the rat: a 850 review of the neuroanatomical literature and comparison with findings from the monkey brain. 851 Hippocampus, 5(5), 390-408.

852 De Curtis, M., \& Paré, D. (2004). The rhinal cortices: a wall of inhibition between the neocortex and the 853 hippocampus. Progress in neurobiology, 74(2), 101-110.

854 Donato, F., Rompani, S. B., \& Caroni, P. (2013). Parvalbumin-expressing basket-cell network plasticity 855 induced by experience regulates adult learning. Nature, 504(7479), 272-276.

856 Feldmeyer, D., Qi, G., Emmenegger, V., Staiger, J.F. (2018) Inhibitory interneurons and their circuit 857 motifs in the many layers of the barrel cortex. Neuroscience, 368:132-151.

858 Gong, S., Zheng, C., Doughty, M. L., Losos, K., Didkovsky, N., Schambra, U. B., . . . Hatten, M. E. 859 (2003). A gene expression atlas of the central nervous system based on bacterial artificial 860 chromosomes. Nature, 425(6961), 917-925.

861 Harris, K. D., \& Shepherd, G. M. (2015). The neocortical circuit: themes and variations. Nature 862 neuroscience, 18(2), 170-181.

863 Hodge, R. D., Bakken, T. E., Miller, J. A., Smith, K. A., Barkan, E. R., Graybuck, L. T., . . Penn, O. 864 (2019). Conserved cell types with divergent features in human versus mouse cortex. Nature, 573(7772), 865 61-68. 
866 Kawaguchi, Y., \& Kubota, Y. (1998). Neurochemical features and synaptic connections of large 867 physiologically-identified GABAergic cells in the rat frontal cortex. Neuroscience, 85(3), 677-701.

868 Kepecs, A., \& Fishell, G. (2014). Interneuron cell types are fit to function. Nature, 505(7483), 318-326.

869 Kim, Y., Yang, G. R., Pradhan, K., Venkataraju, K. U., Bota, M., Del Molino, L. C. G., . . Levine, J. M. 870 (2017). Brain-wide maps reveal stereotyped cell-type-based cortical architecture and subcortical sexual 871 dimorphism. Cell, 171(2), 456-469. e422.

872 Lee, S., Hjerling-Leffler, J., Zagha, E., Fishell, G., \& Rudy, B. (2010). The largest group of superficial 873 neocortical GABAergic interneurons expresses ionotropic serotonin receptors. Journal of Neuroscience, 874 30(50), 16796-16808.

875 Luo, L. (2021). Architectures of neuronal circuits. Science, 373(6559), eabg7285.

$876 \mathrm{Ma}$, Y., Hu, H., Berrebi, A. S., Mathers, P. H., \& Agmon, A. (2006). Distinct subtypes of somatostatin877 containing neocortical interneurons revealed in transgenic mice. Journal of Neuroscience, 26(19), 50698785082.

879 McIntyre, D. C., Kelly, M. E., \& Staines, W. A. (1996). Efferent projections of the anterior perirhinal cortex 880 in the rat. Journal of Comparative Neurology, 369(2), 302-318.

881 Naka, A., Veit, J., Shababo, B., Chance, R. K., Risso, D., Stafford, D., . . . Sridharan, S. (2019). 882 Complementary networks of cortical somatostatin interneurons enforce layer specific control. Elife, 8 , 883 e43696.

884 Nigro, M. J., Hashikawa-Yamasaki, Y., \& Rudy, B. (2018). Diversity and connectivity of layer 5 885 somatostatin-expressing interneurons in the mouse barrel cortex. Journal of Neuroscience, 38(7), 16228861633.

887 Nigro, M. J., Kirikae, H., Kjelsberg, K., Nair, R. R., \& Witter, M. P. (2021). Not all that is gold glitters: PV888 IRES-Cre mouse line shows low efficiency of labeling of parvalbumin interneurons in the perirhinal 889 cortex. Frontiers in Neural Circuits, 15.

890 Overstreet-Wadiche, L., \& McBain, C. J. (2015). Neurogliaform cells in cortical circuits. Nature Reviews 891 Neuroscience, 16(8), 458-468.

892 Pitkänen, A., \& Amaral, D. G. (1993). Distribution of parvalbumin-immunoreactive cells and fibers in the 893 monkey temporal lobe: the hippocampal formation. Journal of Comparative Neurology, 331(1), 37-74.

894 Rudy, B., Fishell, G., Lee, S., \& Hjerling-Leffler, J. (2011). Three groups of interneurons account for 895 nearly $100 \%$ of neocortical GABAergic neurons. Developmental neurobiology, 71(1), 45-61.

896 Scala, F., Kobak, D., Shan, S., Bernaerts, Y., Laturnus, S., Cadwell, C. R., . . Tan, Z. H. (2019). Layer 8974 of mouse neocortex differs in cell types and circuit organization between sensory areas. Nature 898 Communications, 10(1), 1-12.

899 Tamamaki, M., Yanagawa, Y., Tomioka, R., Miyazaki, J., Obata, K., Kaneko, T. (2003) Green 900 fluorescent protein expression and colocalization with calretinin, parvalbumin and somatostatin in the 901 GAD67-GFP knock-in mouse. The Journal of Comparative Neurology, 467:60-79.

902 Taniguchi, H., Lu, J., Huang, Z.J. The spatial and temporal origin of chandelier cells in mouse cortex. 903 Science, 339(6115):70-74.

904 Tasic, B., Yao, Z., Graybuck, L. T., Smith, K. A., Nguyen, T. N., Bertagnolli, D., . . Viswanathan, S. 905 (2018). Shared and distinct transcriptomic cell types across neocortical areas. Nature, 563(7729), 7290678.

907 Tremblay, R., Lee, S., \& Rudy, B. (2016). GABAergic interneurons in the neocortex: from cellular 908 properties to circuits. Neuron, 91(2), 260-292. 
909 Uva, L., Grüschke, S., Biella, G., De Curtis, M., \& Witter, M. P. (2004). Cytoarchitectonic characterization 910 of the parahippocampal region of the guinea pig. Journal of Comparative Neurology, 474(2), 289-303.

911 Van Brederode, J., Helliesen, M., \& Hendrickson, A. (1991). Distribution of the calcium-binding proteins 912 parvalbumin and calbindin-D28k in the sensorimotor cortex of the rat. Neuroscience, 44(1), 157-171.

913 Van Essen, D. C., \& Glasser, M. F. (2018). Parcellating cerebral cortex: how invasive animal studies 914 inform noninvasive mapmaking in humans. Neuron, 99(4), 640-663.

915 Vormstein-Schneider, D., Lin, J. D., Pelkey, K. A., Chittajallu, R., Guo, B., Arias-Garcia, M. A., . . . 916 Stevenson, O. (2020). Viral manipulation of functionally distinct interneurons in mice, non-human 917 primates and humans. Nature neuroscience, 23(12), 1629-1636.

918 Wester, J. C., Mahadevan, V., Rhodes, C. T., Calvigioni, D., Venkatesh, S., Maric, D., ... Petros, T. J. 919 (2019). Neocortical projection neurons instruct inhibitory interneuron circuit development in a lineage920 dependent manner. Neuron, 102(5), 960-975. e966.

921 Whissell, P. D., Cajanding, J. D., Fogel, N., \& Kim, J. C. (2015). Comparative density of CCK-and PV922 GABA cells within the cortex and hippocampus. Frontiers in neuroanatomy, 9, 124.

923 Willems, J. G., Wadman, W. J., \& Cappaert, N. L. (2018). Parvalbumin interneuron mediated 924 feedforward inhibition controls signal output in the deep layers of the perirhinal-entorhinal cortex. 925 Hippocampus, 28(4), 281-296.

926 Witter, M. P., Naber, P. A., van Haeften, T., Machielsen, W. C., Rombouts, S. A., Barkhof, F., ... Lopes 927 da Silva, F. H. (2000). Cortico-hippocampal communication by way of parallel parahippocampal928 subicular pathways. Hippocampus, 10(4), 398-410.

929 Xu, X., Roby, K. D., \& Callaway, E. M. (2010). Immunochemical characterization of inhibitory mouse 930 cortical neurons: three chemically distinct classes of inhibitory cells. Journal of Comparative Neurology, 931 518(3), 389-404.

932 Yao, Z., van Velthoven, C. T., Nguyen, T. N., Goldy, J., Sedeno-Cortes, A. E., Baftizadeh, F., . . . 933 Crichton, K. (2021). A taxonomy of transcriptomic cell types across the isocortex and hippocampal 934 formation. Cell, 184(12), 3222-3241. e3226.

935

936

937

938

939

940

941

942

943

944

945

946

947 

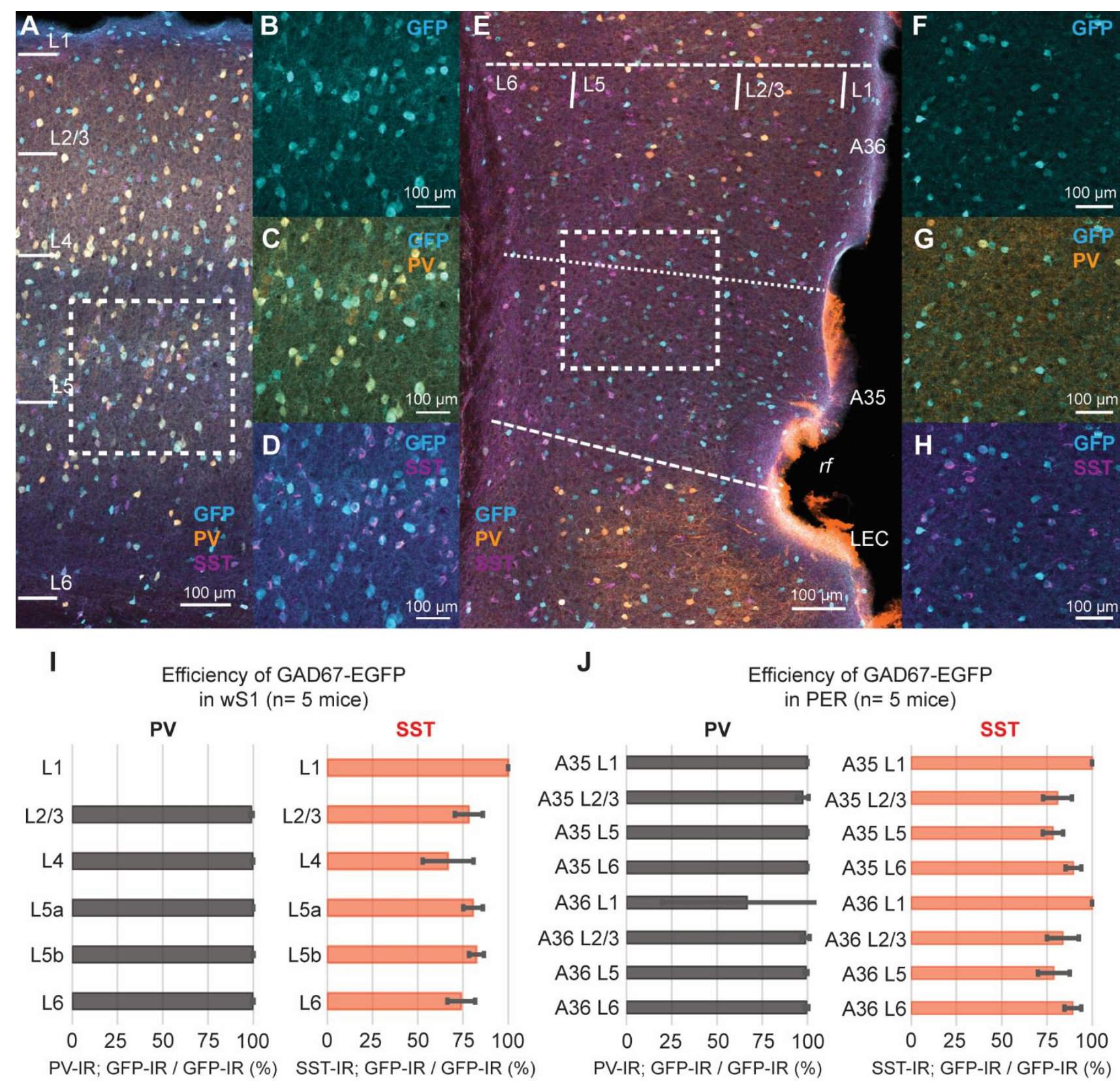

948

949

950

951 Figure 1 - Figure supplement 1. A. Representative confocal stack of immunofluorescence for GFP (turquoise), 952 PV (orange) and SST (purple) in the wS1 of a GAD67-EGFP mouse. B-D. Magnification of the area in the dotted 953 rectangle in A. E. Representative confocal stack of immunofluorescence for GFP (turquoise), PV (orange) and 954 SST (purple) in PER of a GAD67-EGFP mouse. F-H. Magnification of the area in the dotted rectangle in E. I. Bar 955 plots showing the efficiency of the GAD67-EGFP mouse line for PV (black, left) and SST (red, right) in wS1. J. Bar 956 plots showing the efficiency of thee GAD67-EGFP mouse line for PV (black, left) and SST (red, right) in PER. 
962
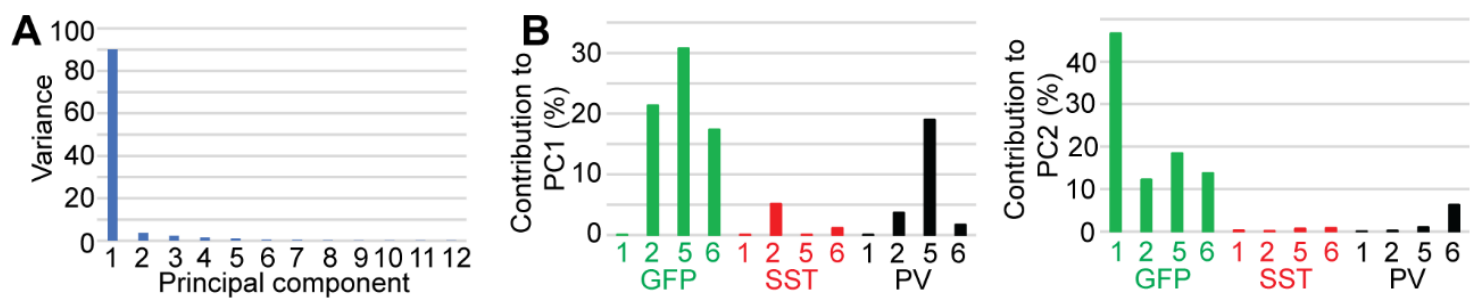

963 Figure 1 - Figure supplement 2. A. Bar plot showing the variance explained by each principal component. B. 964 Bar plots showing the contribution of each variable (i.e., marker density in a given layer) to the first (left) and 965 second (right) principal component.

966

967

968

969

970

971

A

PV: A35, A36, wS1

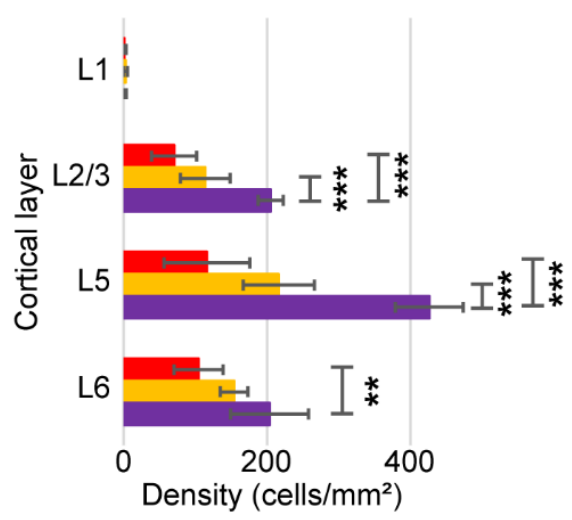

B

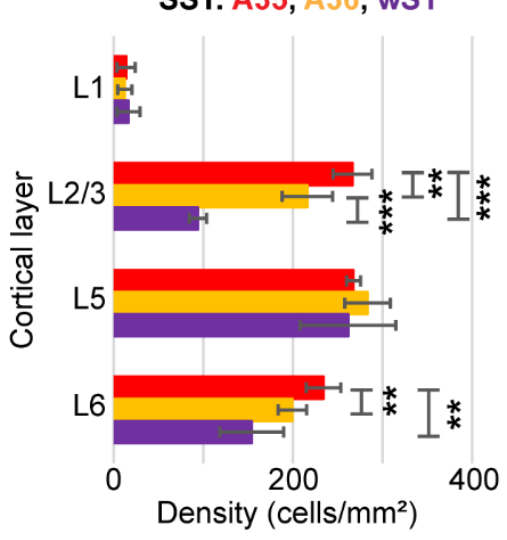

C

GFP: A35, A36, wS1

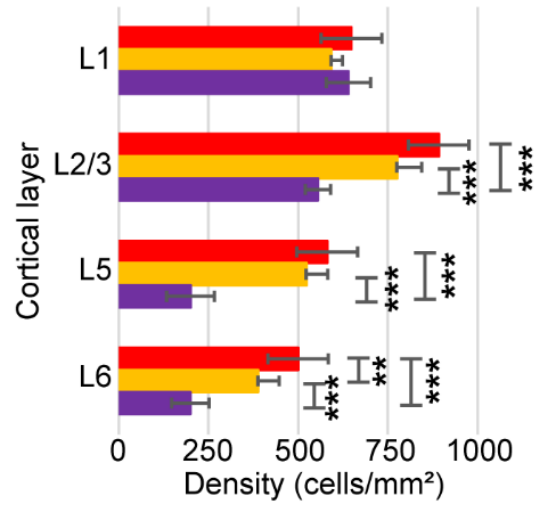

973

974 Figure 1 - Figure supplement 3. A. Bar plot showing the statistical comparison of the density of PV-IR neurons 975 in the GAD67-EGFP mouse line ( $n=5$ mice): $L 1(F=2.06, d f=2, p=0.17$, One-way ANOVA); $L 2 / 3(F=28.54, d f=$ 976 2, $p=2.75 E-5$, One-way ANOVA; A35 vs wS1, $p=3.16 E-5$, Bonferroni correction; A36 vs wS1= 0.0007, Bonferroni 977 correction); $\mathrm{L} 5(\mathrm{~F}=45.5, \mathrm{df}=2, \mathrm{p}=2.5 \mathrm{E}-6$, One-way ANOVA; A35 vs wS1, $\mathrm{p}=2.37 \mathrm{E}-5$, Bonferroni correction; A36 978 vs wS1, $p=0.0001$, Bonferroni correction); L6 ( $F=8.17, d f=2, p=0.006$, One-way ANOVA; A35 vs wS1, $p=0.011$, 979 Bonferroni correction). B. Bar plot showing the statistical comparison of the density of SST-IR neurons in the 980 GAD67-EGFP mouse line: $L 1(F=0.21, d f=2, p=0.82$, One-way ANOVA); $L 2 / 3(F=87.06, d f=2, p=7.18 E-8$, One981 way ANOVA; A35 vs A36, $p=0.014$, Bonferroni correction; A35 vs wS1, $p=6.85 E-6$, Bonferroni correction; A36 vs 982 wS1, $p=0.0003$, Bonferroni correction); L5 ( $F=0.53, d f=2, p=0.6$, One-way ANOVA); L6 $(F=12.83, d f=2, p=$ 983 0.001, One-way ANOVA; A35 vs A36, $p=0.015$, Bonferroni correction; A35 vs wS1, $p=0.004$, Bonferroni 984 correction). C. Bar plot showing the statistical comparison of the density of GFP-IR/-PV-SST neurons in the 985 GAD67-EGFP mouse line: $L 1(F=1.61, \mathrm{df}=2, \mathrm{p}=0.24$, One-way $A N O V A) ; \mathrm{L} 2 / 3(\mathrm{~F}=51.43, \mathrm{df}=2, \mathrm{p}=1.3 \mathrm{E}-6$, One986 way ANOVA; A35 vs wS1, $p=6.04 \mathrm{E}-6$, Bonferroni correction; A36 vs wS1, $p=0.0007$, Bonferroni correction); L5 $987(\mathrm{~F}=56.88, \mathrm{df}=2, \mathrm{p}=7.55 \mathrm{E}-7$, One-way ANOVA; $\mathrm{A} 35$ vs wS1, $\mathrm{p}=1.21 \mathrm{E}-5$, Bonferroni correction; $\mathrm{A} 36 \mathrm{vs}$ wS1, $\mathrm{p}=$ 988 4.16E-5, Bonferroni correction); $\mathrm{L} 6(\mathrm{~F}=38.09, \mathrm{df}=2, \mathrm{p}=6.35 \mathrm{E}-6$, One-way ANOVA; A35 vs $A 36, p=0.014$, 989 Bonferroni correction; A35 vs wS1, $p=1.99 \mathrm{E}-5$, Bonferroni correction; A36 vs wS1, $p=0.0007$, Bonferroni 990 correction).

991

992

993

994 

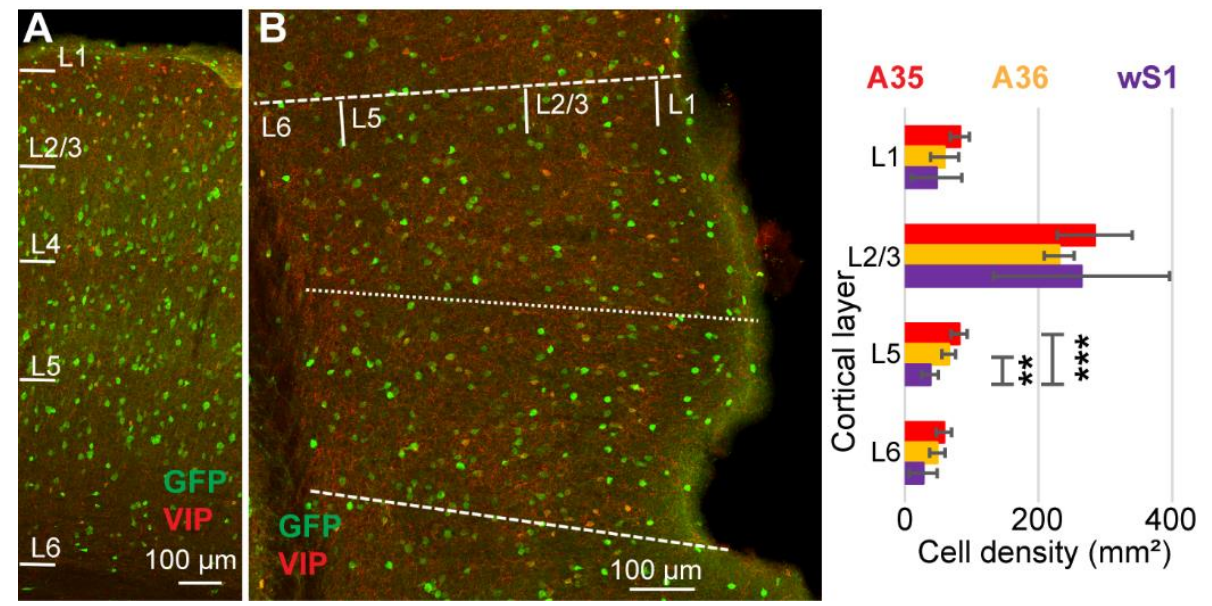

996 Figure 2 - Figure 2 supplement 1. A. Representative confocal stack of immunofluorescence for GFP (green) 997 and VIP (red) in wS1 of the GAD67-EGFP mouse line. B. Representative confocal stack of immunofluorescence 998 for GFP (green) and VIP (red) in PER of the GAD-EGFP mouse line. C. Bar plot showing the statistical comparison 999 of the density of VIP-IR neurons across layers of A35 (red), A36 (yellow) and wS1 (purple) ( $n=5$ mice): L1 (F= 1000 2.34, $\mathrm{df}=2, \mathrm{p}=0.14$, One-way ANOVA); L2/3 $(\mathrm{F}=0.51, \mathrm{df}=2, \mathrm{p}=0.61$, One-way ANOVA $) ; \mathrm{L} 5(\mathrm{~F}=17.76, \mathrm{df}=2, \mathrm{p}=$ 1001 0.0003, One-way ANOVA; A35 vs wS1, $p=0.0005$, Bonferroni correction; A36 vs wS1, $p=0.005$, Bonferroni 1002 correction); L6 ( $F=5.22, d f=2, p=0.02$, One-way ANOVA).

1003

1004

1005

1006

1007

1008

1009

1010

1011

1012

1013

1014

1015

1016

1017

1018

1019

1020

1021

1022

1023

1024

1025

1026

1027

1028

1029

1030

1031

1032

1033 


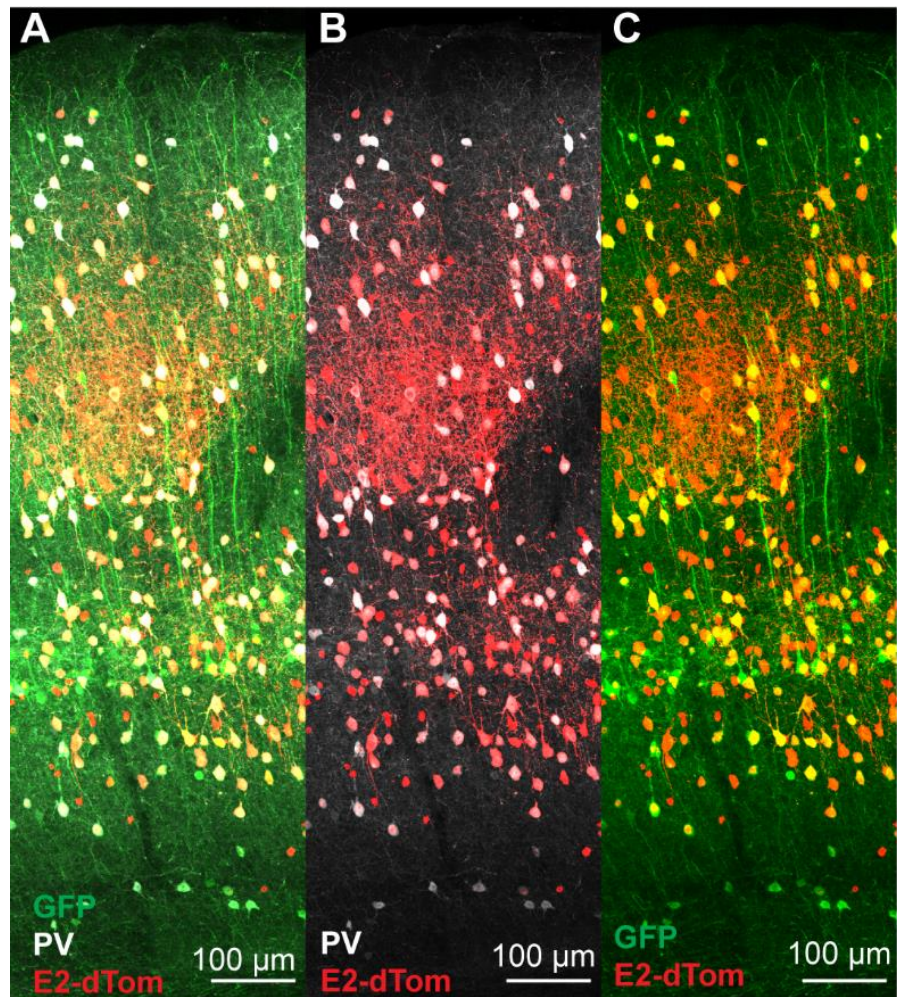

D

100

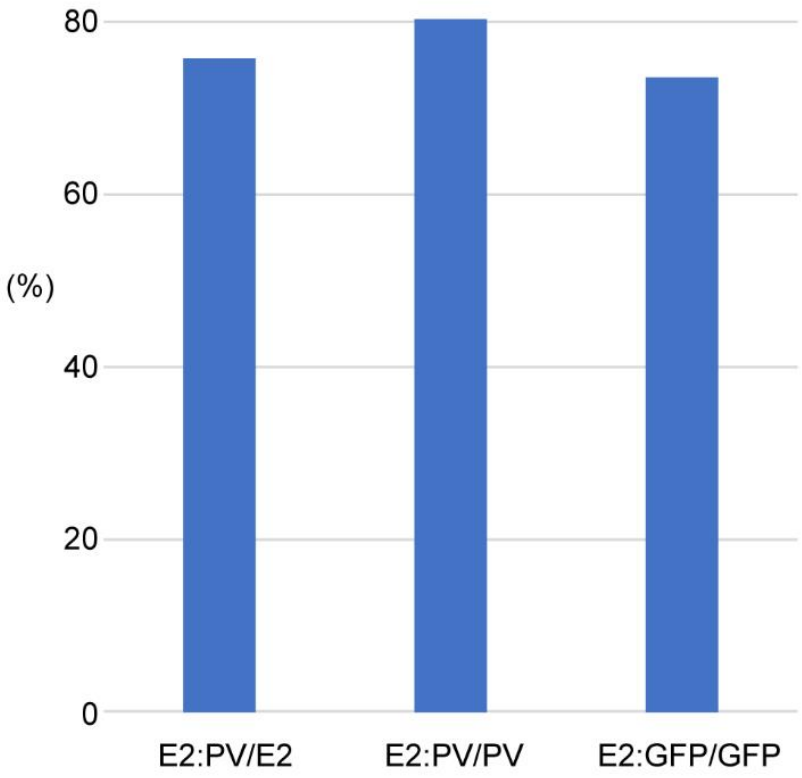

$$
\text { E }
$$

F

G

H

100

80

60

(\%)

40
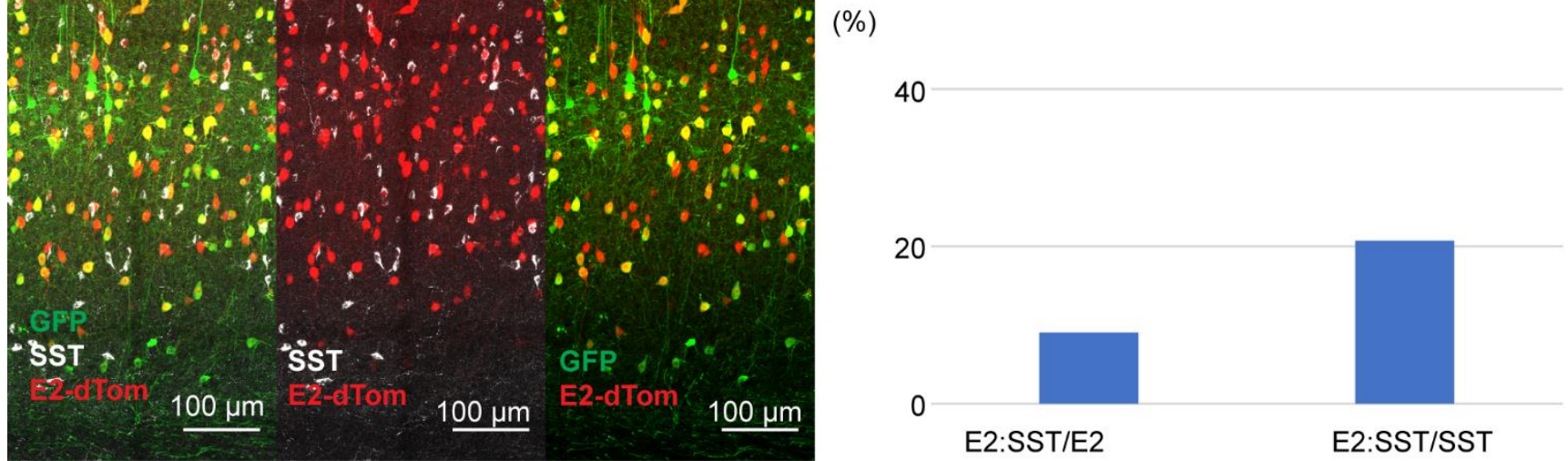

40

E2:SST/SST

1035 Figure 4 - Figure supplement 1. A-C. Representative confocal stacks of immunofluorescence for GFP (green), 1036 PV (white) and dTom (red) in a PV-IRES-Cre mouse injected with the S5E2-dTom virus in wS1. D. Bar plot showing 1037 the specificity (E2:PV/E2), and efficiency (E2:PV/PV and E2:GFP/GFP) of the S5E2-dTom virus in wS1 ( $n=1$ 1038 mouse). E-G. Representative confocal stacks of immunofluorescence for GFP (green), SST (white) and dTom 1039 (red) in PV-IRES-Cre mice injected with the S5E2-dTom virus in wS1. H. Bar plot showing the percent of neurons 1040 expressing dTomato (E2) that co-express SST (E2:SST/E2), and the percent of SST neurons labeled by the S5E21041 dTom virus (E2:SST/SST) $(n=1$ mouse). 

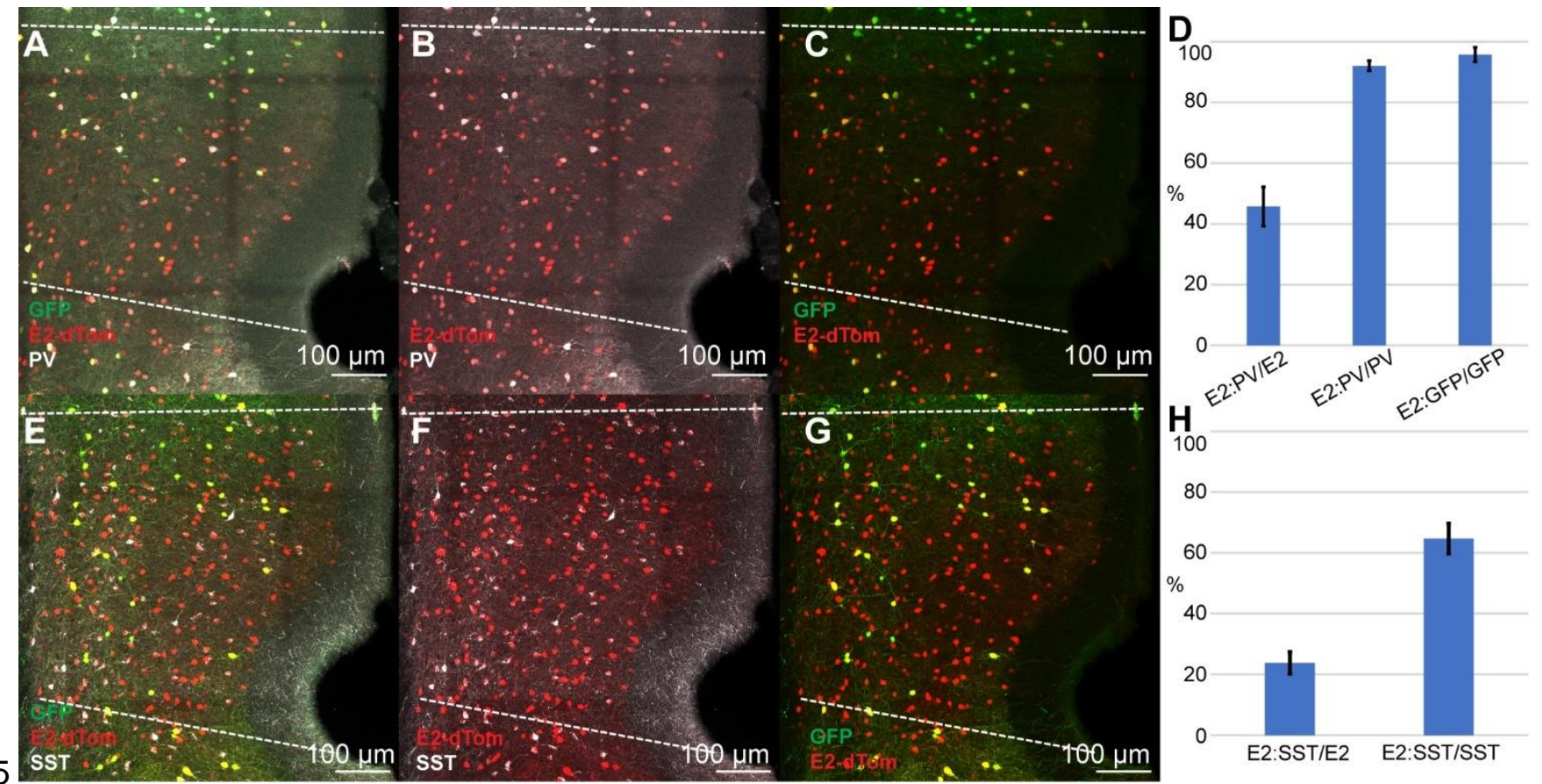

1046

1047 Figure 4 - Figure supplement 2. A-C. Representative confocal stacks of immunofluorescence for GFP (green), 1048 E2-dTom (red) and PV (white) in PV-IRES-Cre mice injected with the S5E2-dTom virus in PER. D. Bar plot showing 1049 the specificity (E2:PV/E2), and efficiency (E2:PV/PV and E2:GFP/GFP) of the S5E2-dTom virus in PER ( $n=2$ 1050 mice). E-G. Representative confocal stacks of immunofluorescence for GFP (green), SST (white) and dTom (red) 1051 in PV-IRES-Cre mice injected with the S5E2-dTom virus in PER. H. Bar plot showing the percent of neurons 1052 expressing dTomato (E2) that co-express SST (E2:SST/E2), and the percent of SST neurons labeled by the S5E21053 dTom virus (E2:SST/SST) ( $n=2$ mice).

1054

1055

1056

1057
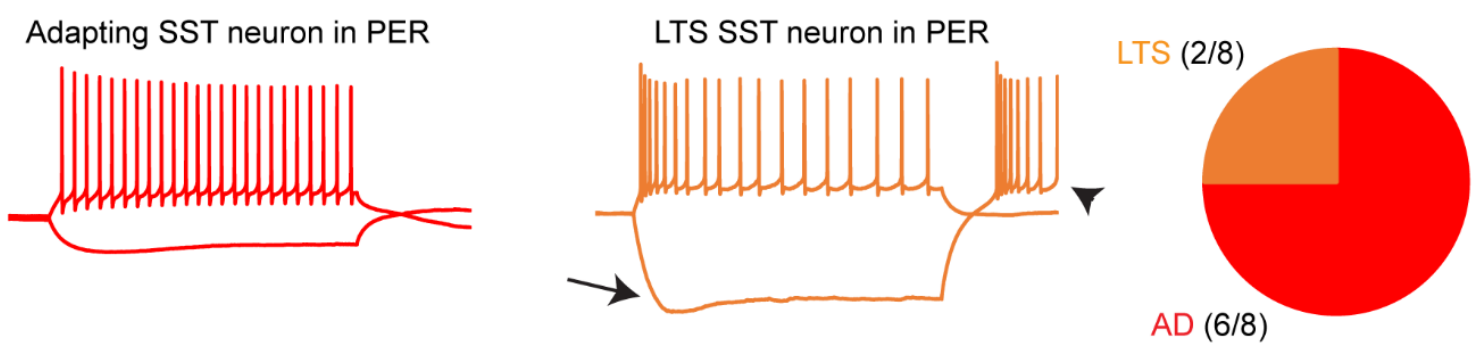

1058

1059

1060

1061 Figure 5 - Figure supplement 1. Representative voltage responses of an adapting (red) and a LTS (orange) 1062 neurons recorded in the SST-Cre mouse line and labeled with tdTomato. The pie chart shows the percentage of 1063 adapting (red) and LTS (orange) neurons in the SST-Cre line.

1064 